\title{
Existence, Uniqueness, and Convergence of optimal control problems associated with Parabolic variational inequalities of the second kind
}

\author{
Mahdi Boukrouche* Domingo A. Tarzia ${ }^{\dagger}$
}

\begin{abstract}
Let $u_{g}$ the unique solution of a parabolic variational inequality of second kind, with a given $g$. Using a regularization method, we prove, for all $g_{1}$ and $g_{2}$, a monotony property between $\mu u_{g_{1}}+(1-\mu) u_{g_{2}}$ and $u_{\mu g_{1}+(1-\mu) g_{2}}$ for $\mu \in[0,1]$. This allowed us to prove the existence and uniqueness results to a family of optimal control problems over $g$ for each heat transfer coefficient $h>0$, associated to the Newton law, and of another optimal control problem associated to a Dirichlet boundary condition. We prove also, when $h \rightarrow+\infty$, the strong convergence of the optimal controls and states associated to this family of optimal control problems with the Newton law to that of the optimal control problem associated to a Dirichlet boundary condition.

Keywords: Parabolic variational inequalities of the second kind, convex combination of solutions, monotony property, regularization method, dependency of the solutions on the data, strict convexity of cost functional, optimal control problems.

2000AMS Subject Classification 35R35, 35B37, 35K85, 49J20, 49K20.
\end{abstract}

Short title : Controls for parabolic variational inequalities

\section{Introduction}

Let consider the following problem governed by the parabolic variational inequality

$$
\langle\dot{u}(t), v-u(t)\rangle+a(u(t), v-u(t))+\Phi(v)-\Phi(u(t)) \geq<g(t), v-u(t)>\quad \forall v \in K,
$$

a.e. $t \in] 0, T[$, with the initial condition

$$
u(0)=u_{b}
$$

where, $a$ is a symmetric continuous and coercive bilinear form on the Hilbert space $V \times V$, $\Phi$ is a proper and convex function from $V$ into $\mathbb{R}$ and is lower semi-continuous for the weak topology on $V,\langle\cdot, \cdot\rangle$ denotes the duality brackets between $V^{\prime}$ and $V, K$ is a closed convex non-empty subset of $V, u_{b}$ is an initial value in another Hilbert space $H$ with $V$ being densely and continuously imbedded in $H$, and $g$ is a given function in the space $L^{2}\left(0, T, V^{\prime}\right)$. It is well known [17, 18, 21, 22 that, there exists a unique solution

$$
u \in \mathcal{C}(0, T, H) \cap L^{2}(0, T, V) \quad \text { with } \quad \dot{u}=\frac{\partial u}{\partial t} \in L^{2}(0, T, H)
$$

* Lyon University, F-42023 Saint-Etienne, Laboratory of Mathematics, University of Saint-Etienne, LaMUSE EA-3989, 23 Docteur Paul Michelon 42023 Saint-Etienne Cedex 2, France. Fax: +33 47748 51 53, Phone +33 4774815 35, E-mail: Mahdi.Boukrouche@univ-st-etienne.fr.

${ }^{\dagger}$ Departamento de Matemática-CONICET, FCE, Univ. Austral, Paraguay 1950, S2000FZF Rosario, Argentina. Fax: +54 341522 3001, Phone +54 341522 3093, E-mail: DTarzia@austral.edu.ar 
to (1.1)-(1.2). So we can consider $g \mapsto u_{g}$ as a function from $L^{2}(0, T, H)$ to $\mathcal{C}(0, T, H) \cap$ $L^{2}(0, T, V)$. Then we can consider [26, 27, 34] the cost functional $J$ defined by

$$
J(g)=\frac{1}{2}\left\|u_{g}\right\|_{L^{2}(0, T, H)}^{2}+\frac{M}{2}\|g\|_{L^{2}(0, T, H)}^{2},
$$

where $M$ is a positive constant, and $u_{g}$ is the unique solution to (1.1)-(1.2), corresponding to the control $g$. One of our main purposes is to prove the existence and uniqueness of the optimal control problem

$$
\text { Find } g_{o p} \in L^{2}(0, T, H) \text { such that } J\left(g_{o p}\right)=\min _{g \in L^{2}(0, T, H)} J(g) \text {. }
$$

This can be reached if we prove the strictly convexity of the cost functional $J$, which follows (see Theorem 3.1) from the following monotony property : for any two control $g_{1}$ and $g_{2}$ in $L^{2}(0, T, H)$,

$$
u_{4}(\mu) \leq u_{3}(\mu) \quad \forall \mu \in[0,1]
$$

where

$$
u_{3}(\mu)=\mu u_{1}+(1-\mu) u_{2}, \quad u_{4}(\mu)=u_{g_{3}(\mu)}, \quad \text { with } \quad g_{3}(\mu)=\mu g_{1}+(1-\mu) g_{2} .
$$

In Section 2, we establish first in Theorem 2.2, the error estimate between $u_{3}(\mu)$ and $u_{4}(\mu)$. This result generalizes our previous result obtained in [16] for the elliptic variational inequalities. We deduce in Corollary 2.3 a condition on the data to get $u_{3}(\mu)=u_{4}(\mu)$ for all $\mu \in[0,1]$. Then we assume, that the convex $K$ is a subset of $V=H^{1}(\Omega)$ and consider the parabolic variational problems $(P)$ and $\left(P_{h}\right)$. So, using a regularization method, we prove in Theorem 2.5 this monotony property (1.5) , for the solutions of the two problems $(P)$ and $\left(P_{h}\right)$. This result with a new proof and simplified, generalizes that obtained by [29] for elliptic variational inequalities. In Subsection 2.1 we also obtain some properties of dependency solutions based on the data $g$ and on a positive parameter $h$ for the parabolic variational inequalities (1.1) and (2.1), see Propositions 2.6, 2.7 and 2.8.

In Section 3, we consider the family of distributed optimal control problems $\left(P_{h}\right)_{h>0}$,

$$
\text { Find } g_{o p_{h}} \in L^{2}(0, T, H) \quad \text { such that } \quad J\left(g_{o p_{h}}\right)=\min _{g \in L^{2}(0, T, H)} J_{h}(g),
$$

with the cost functional

$$
J_{h}(g)=\frac{1}{2}\left\|u_{g_{h}}\right\|_{L^{2}(0, T, H)}^{2}+\frac{M}{2}\|g\|_{L^{2}(0, T, H)}^{2},
$$

where $u_{g_{h}}$ is the unique solution of (2.1)-(1.2), corresponding to the control $g$ for each $h>0$, and the distributed optimal control problems

$$
\text { Find } g_{o p} \in L^{2}(0, T, H) \quad \text { such that } \quad J\left(g_{o p}\right)=\min _{g \in L^{2}(0, T, H)} J(g) \text {, }
$$

with the cost functional (1.3) where $u_{g}$ is the unique solution to (1.1)-(1.2), corresponding to the control $g$. Using Theorem 2.5 with its crucial property of monotony (1.5), we prove the strict convexity of the cost functional (1.3) and also of the cost functional (1.8), associated to the problems (1.9) and (1.7) respectively. Then, the existence and uniqueness of solutions to the optimal controls problems (1.9) and (1.7) follows from [27].

In general see for example [20] the relevant physical condition, to impose on the boundary, is Newton's law, or Robin's law, and not Dirichlet's. Therefore, the objective of this 
work is to approximate the optimal control problem (1.9), where the state is the solution to parabolic variational problem (1.1)-(1.2) associated with the Dirichlet condition (2.2), by a family indexed by a factor $h$ of optimal control problems (2.1)-(1.2), where states are the solutions to parabolic variational problems, associated with the boundary condition of Newton (2.3). Moreover, from a numerical analysis point of view it maybe preferable to consider approximating Neumann problems in all space $V$ (see (2.1)-(1.2)), with parameter $h$, rather than the Dirichlet problem in a subset of the space $V$ (see (1.1)-(1.2)). So the asymptotic behavior can be considered very important in the optimal control.

In the last subsection 3.1, which is also the goal of our paper, we prove that the optimal control $g_{o p_{h}}$ (unique solution of the optimization problem (1.7)) and its corresponding state $u_{g_{o p_{h} h}}$ (the unique solution of the parabolic variational problem (2.1)-(1.2)) for each $h>1$, are strongly convergent to $g_{o p}$ (the unique solution of the optimization problem (1.9) ), and $u_{g_{o p}}$ (the unique solution of the parabolic variational problem (1.1)-(1.2)) in $L^{2}([0, T] \times \Omega)$ and $L^{2}\left(0, T, H^{1}(\Omega)\right)$ respectively when $h \rightarrow+\infty$.

This paper generalizes the results obtained in [23], for elliptic variational equalities, and in [28] for parabolic variational equalities, to the case of parabolic variational inequalities of second kind. Various problems with distributed optimal control, associated with elliptic variational inequalities are given see for example [1, 4], [7]-[9], [19, 25], [29]-[31], [39] and for the parabolic case see for example [2, 4, 5], [10]-[12], [32, 33], [35].

\section{On the property of monotony}

As we can not prove the property of monotony (1.5) for any convex set $K$. Let $\Omega$ a bounded open set in $\mathbb{R}^{N}$ with smooth boundary $\partial \Omega=\Gamma_{1} \cup \Gamma_{2}$. We assume that $\Gamma_{1} \cap \Gamma_{2}=\varnothing$, and meas $\left(\Gamma_{1}\right)>0$. Let $H=L^{2}(\Omega), V=H^{1}(\Omega)$. We can prove the property of monotony (1.5) for any convex subset of $V$. Let

$$
K=\left\{v \in V: \quad v_{\mid \Gamma_{1}}=0\right\}, \quad \text { and } \quad K_{b}=\left\{v \in V: \quad v_{\mid \Gamma_{1}}=b\right\} .
$$

So we consider the following variational problems with such convex subset.

Problem $(P)$ Let given $b \in L^{2}(] 0, T\left[\times \Gamma_{1}\right), g \in L^{2}(0, T, H)$ and $q \in L^{2}(] 0, T\left[\times \Gamma_{2}\right), q>0$. Find $u$ in $\mathcal{C}([0, T], H) \cap L^{2}\left(0, T, K_{b}\right)$ solution of the parabolic problem (1.1), where $\langle\cdot, \cdot\rangle$ is only the scalar product $(\cdot, \cdot)$ in $H$, with the initial condition (1.2), and $\Phi(v)=\int_{\Gamma_{2}} q|v| d s$.

Problem $\left(P_{h}\right)$ Let given $b \in L^{2}(] 0, T\left[\times \Gamma_{1}\right), g \in L^{2}(0, T, H)$ and $q \in L^{2}(] 0, T\left[\times \Gamma_{2}\right), q>0$. For all coefficient $h>0$, find $u \in \mathcal{C}(0, T, H) \cap L^{2}(0, T, V)$ solution of the parabolic variational inequality

$$
\begin{array}{r}
\langle\dot{u}(t), v-u(t)\rangle+a_{h}(u(t), v-u(t))+ \\
+h(v)-\Phi(u(t)) \geq(g(t), v-u(t)) \\
+h \int_{\Gamma_{1}} b(t)(v-u(t)) d s \quad \forall v \in V,
\end{array}
$$

and the initial condition (1.2), where $a_{h}(u, v)=a(u, v)+h \int_{\Gamma_{1}} u v d s$.

It is easy to see that the problem $(P)$ is with the Dirichlet condition

$$
\left.u=b \quad \text { on } \quad \Gamma_{1} \times\right] 0, T[
$$

and the problem $\left(P_{h}\right)$ is with the following Newton-Robin's type condition

$$
\left.-\frac{\partial u}{\partial n}=h(u-b) \quad \text { on } \quad \Gamma_{1} \times\right] 0, T[.
$$


where $n$ is the exterior unit vector normal to the boundary. The integal on $\Gamma_{2}$ in the expression of $\Phi$ comes from the Tresca boundary condition (see [13]-[15], [22]) with $q$ is the Tresca friction coefficient on $\Gamma_{2}$. Note that only for the proof of Theorem 2.5 we have need to specify an expression of the functional $\Phi$.

By assumption there exists $\lambda>0$ such that $\lambda\|v\|_{V}^{2} \leq a(v, v) \quad \forall v \in V$. Moreover, it follows from [36, 37] that there exists $\lambda_{1}>0$ such that

$$
a_{h}(v, v) \geq \lambda_{h}\|v\|_{V}^{2} \quad \forall v \in V, \quad \text { with } \lambda_{h}=\lambda_{1} \min \{1, h\}
$$

so $a_{h}$ is a bilinear, continuous, symmetric and coercive form on $V$. So there exists an unique solution to each of the two problems $(P)$ and $\left(P_{h}\right)$.

We recall that $u_{g}$ is the unique solution of the parabolic variational problem $(P)$, corresponding to the control $g \in L^{2}(0, T, H)$, and also that $u_{g_{h}}$ is the unique solution of the parabolic variational problem $\left(P_{h}\right)$, corresponding to the control $g \in L^{2}(0, T, H)$.

Proposition 2.1. Assume that $g \geq 0$ in $\Omega \times] 0, T\left[, b \geq 0\right.$ on $\left.\Gamma_{1} \times\right] 0, T\left[, u_{b} \geq 0\right.$ in $\Omega$. Then as $q>0$, we have $u_{g} \geq 0$. Assuming again that $h>0$, then $u_{g_{h}} \geq 0$ in $\left.\Omega \times\right] 0, T$.

Proof. For $u=u_{g_{h}}$, it is enough to take $v=u^{+}$in (2.1), to get

$$
\begin{gathered}
\left\|u^{-}(T)\right\|_{L^{2}(\Omega)}^{2}+\lambda \int_{0}^{T}\left\|u^{-}(t)\right\|_{V}^{2} d t+h \int_{0}^{T} \int_{\Gamma_{1}}\left(u^{-}(t)\right)^{2} d s d t+\leq-\int_{0}^{T}\left(g(t), u^{-}(t)\right) d t \\
-\int_{0}^{T} \int_{\Gamma_{2}} q\left(|u(t)|-\left|u^{+}(t)\right|\right) d s d t-h \int_{0}^{T} \int_{\Gamma_{1}} b(t) u^{-}(t) d s d t+\left\|u^{-}(0)\right\|_{L^{2}(\Omega)}^{2}
\end{gathered}
$$

so the result follows.

Theorem 2.2. Let $u_{1}$ and $u_{2}$ be two solutions of the parabolic variational inequality (1.1) with the same initial condition, and corresponding to the two control $g_{1}$ and $g_{2}$ respectively. We have the following estimate

$$
\begin{array}{r}
\frac{1}{2}\left\|u_{4}(\mu)-u_{3}(\mu)\right\|_{L^{\infty}(0, T, H)}^{2}+\lambda\left\|u_{4}(\mu)-u_{3}(\mu)\right\|_{L^{2}(0, T, V)}^{2}+\mu \mathcal{I}_{14}(\mu)(T)+(1-\mu) \mathcal{I}_{24}(\mu)(T) \\
\quad+\mu \Phi\left(u_{1}\right)+(1-\mu) \Phi\left(u_{2}\right)-\Phi\left(u_{3}(\mu)\right) \leq \mu(1-\mu)\left(\mathcal{A}\left(T, g_{1}\right)+\mathcal{B}\left(T, g_{2}\right)\right) \quad \forall \mu \in[0,1],
\end{array}
$$

where

$$
\begin{gathered}
\mathcal{I}_{j 4}(\mu)(T)=\int_{0}^{T} I_{j 4}(\mu)(t) d t \quad \text { for } j=1,2, \quad \mathcal{A}\left(T, g_{1}\right)=\int_{0}^{T} \alpha(t) d t, \quad \mathcal{B}\left(T, g_{2}\right)=\int_{0}^{T} \beta(t) d t, \\
I_{j 4}(\mu)=\left\langle\dot{u}_{j}, u_{4}(\mu)-u_{j}\right\rangle+a\left(u_{j}, u_{4}(\mu)-u_{j}\right)+\Phi\left(u_{4}(\mu)\right)-\Phi\left(u_{j}\right)-\left\langle g_{j}, u_{4}(\mu)-u_{j}\right\rangle \geq 0 \\
\alpha=\left\langle\dot{u}_{1}, u_{2}-u_{1}\right\rangle+a\left(u_{1}, u_{2}-u_{1}\right)+\Phi\left(u_{2}\right)-\Phi\left(u_{1}\right)-\left\langle g_{1}, u_{2}-u_{1}\right\rangle \geq 0 \\
\beta=\left\langle\dot{u}_{2}, u_{1}-u_{2}\right\rangle+a\left(u_{2}, u_{1}-u_{2}\right)+\Phi\left(u_{1}\right)-\Phi\left(u_{2}\right)-\left\langle g_{2}, u_{1}-u_{2}\right\rangle \geq 0 .
\end{gathered}
$$

Proof. As $u_{3}(\mu)(t) \in K$ so with $v=u_{3}(\mu)(t)$, in the variational inequality (1.1) where $u=u_{4}(\mu)$ and $g=g_{3}(\mu)$, we obtain

$$
\begin{array}{r}
\left\langle\dot{u}_{4}(\mu), u_{3}(\mu)-u_{4}(\mu)\right\rangle+a\left(u_{4}(\mu), u_{3}(\mu)-u_{4}(\mu)\right)+\Phi\left(u_{3}(\mu)\right)-\Phi\left(u_{4}(\mu)\right) \\
\left.\geq\left\langle g_{3}(\mu), u_{3}(\mu)-u_{4}(\mu)\right\rangle \text { a.e.t } \in\right] 0, T[
\end{array}
$$


then

$$
\begin{array}{r}
\left\langle\dot{u}_{4}(\mu)-\dot{u}_{3}(\mu), u_{4}(\mu)-u_{3}(\mu)\right\rangle+a\left(u_{4}(\mu)-u_{3}(\mu), u_{4}(\mu)-u_{3}(\mu)\right) \\
\leq\left\langle\dot{u}_{3}(\mu), u_{3}(\mu)-u_{4}(\mu)\right\rangle+a\left(u_{3}(\mu), u_{3}(\mu)-u_{4}(\mu)\right) \\
\left.+\Phi\left(u_{3}(\mu)\right)-\Phi\left(u_{4}(\mu)(t)\right)-\left\langle g_{3}(\mu), u_{3}(\mu)-u_{4}(\mu)\right\rangle \quad \text { a.e. } t \in\right] 0, T[
\end{array}
$$

thus

$$
\begin{array}{r}
\frac{1}{2} \frac{\partial}{\partial t}\left(\left\|u_{4}(\mu)-u_{3}(\mu)\right\|_{H}^{2}\right)+\lambda\left\|u_{4}(\mu)-u_{3}(\mu)\right\|_{V}^{2} \leq\left\langle\dot{u}_{3}(\mu), u_{3}(\mu)-u_{4}(\mu)\right\rangle \\
+a\left(u_{3}(\mu), u_{3}(\mu)-u_{4}(\mu)\right)+\Phi\left(u_{3}(\mu)\right)-\Phi\left(u_{4}(\mu)\right) \\
\left.-\left\langle g_{3}(\mu), u_{3}(\mu)-u_{4}(\mu)\right\rangle, \quad \text { a.e.t } \in\right] 0, T[
\end{array}
$$

using that $u_{3}(\mu)=\mu\left(u_{1}-u_{2}\right)+u_{2}, g_{3}(\mu)=\mu\left(g_{1}-g_{2}\right)+g_{2}$ we get

$$
\begin{aligned}
\frac{1}{2} \frac{\partial}{\partial t}\left(\left\|u_{4}(\mu)-u_{3}(\mu)\right\|_{H}^{2}\right) & +\lambda\left\|u_{4}(\mu)-u_{3}(\mu)\right\|_{V}^{2}+\mu \Phi\left(u_{1}\right)+(1-\mu) \Phi\left(u_{2}\right)-\Phi\left(u_{3}(\mu)\right. \\
\leq & \left.\mu(1-\mu)(\alpha+\beta)-\mu I_{14}(\mu)-(1-\mu) I_{24}(\mu) \quad \text { a.e. } \in\right] 0, T[,
\end{aligned}
$$

so by integration between $t=0$ and $t=T$, we deduce the required result.

Corollary 2.3. From Theorem 2.2 we get a.e. $t \in[0, T]$

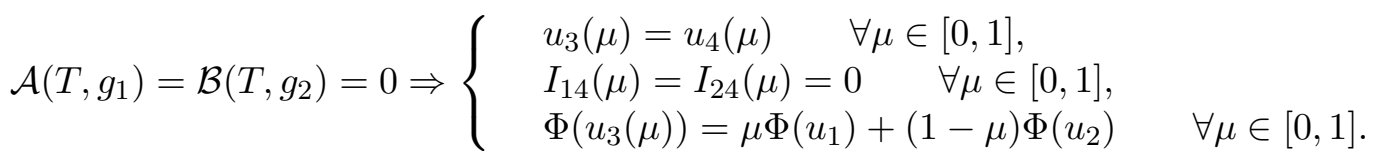

Lemma 2.4. Let $u_{1}$ and $u_{2}$ be two solutions of the parabolic variational inequality of second kind (1.1) with respectively as second member $g_{1}$ and $g_{2}$, then we get

$$
\left\|u_{1}-u_{2}\right\|_{L^{\infty}(0, T, H)}^{2}+\lambda\left\|u_{1}-u_{2}\right\|_{L^{2}(0, T, V)}^{2} \leq \frac{1}{\lambda}\left\|g_{1}-g_{2}\right\|_{L^{2}\left(0, T, V^{\prime}\right)}^{2},
$$

Where $\lambda$ is the coerciveness constant of the biliear form a.

Proof. Taking $v=u_{2}$ in (1.1) where $u=u_{1}$ and $g=g_{1}$; then $v=u_{1}$ in (1.1) where $u=u_{2}$ and $g=g_{2}$, so by addition (2.7) holds.

We generalize now in our case the result on a monotony property, obtained by [29] for the elliptic variational inequality. This theorem is the cornestone to prove the strict convexity of the cost functional $J$ defined in Problem (1.9) and the cost functional $J_{h}$ defined in Problem (1.7). Remark first that with the duality bracks $<\cdot, \cdot\rangle$ defined by

$$
<g(t), \varphi>=(g(t), \varphi)+h \int_{\Gamma_{1}} b(t) \varphi d s
$$

(2.1) leads to (1.1). We prove the following theorem for $\Phi$ such that $\Phi(v)=\int_{\Gamma_{2}} q|v| d s$.

Theorem 2.5. For any two control $g_{1}$ and $g_{2}$ in $L^{2}(0, T, H)$, it holds that

$$
u_{4}(\mu) \leq u_{3}(\mu) \quad \text { in } \quad \Omega \times[0, T], \quad \forall \mu \in[0,1] .
$$

Here $u_{4}(\mu)=u_{\mu g_{1}+(1-\mu) g_{2}}, u_{3}(\mu)=\mu u_{g_{1}}+(1-\mu) u_{g_{2}}, u_{1}=u_{g_{1}}$ and $u_{2}=u_{g_{2}}$ are the unique solutions of the variational problem $P$, with $g=g_{1}$ and $g=g_{2}$ respectively, and for the same $q$, and the same initial condition (1.2). Moreover, it holds also that

$$
u_{h 4}(\mu) \leq u_{h 3}(\mu) \quad \text { in } \quad \Omega \times[0, T], \quad \forall \mu \in[0,1] .
$$


Here $u_{4 h}(\mu)=u_{\mu g_{1 h}+(1-\mu) g_{2 h}}, u_{3 h}(\mu)=\mu u_{g_{1 h}}+(1-\mu) u_{g_{2 h}}, u_{1 h}=u_{g_{1 h}}$ and $u_{h 2}=u_{g_{h 2}}$ are the unique solutions of the variational problem $P_{h}$, with $g=g_{1}$ and $g=g_{2}$ respectively, and for the same $q, h, b$ and the same initial condition (1.2).

Proof. The main difficulty, to prove this result comes from the fact that the functional $\Phi$ is not differentiable. To overcome this difficulty, we use the regularization method and consider for $\varepsilon>0$ the following approach of $\Phi$

$$
\Phi_{\varepsilon}(v)=\int_{\Gamma_{2}} q \sqrt{\varepsilon^{2}+|v|^{2}} d s, \quad \forall v \in V,
$$

which is Gateaux differentiable, with

$$
\left\langle\Phi_{\varepsilon}^{\prime}(w), v\right\rangle=\int_{\Gamma_{2}} \frac{q w v}{\sqrt{\varepsilon^{2}+|w|^{2}}} d s \quad \forall(w, v) \in V^{2} .
$$

Let $u^{\varepsilon}$ be the unique solution of the variational inequality

$$
\begin{array}{r}
\left\langle\dot{u}^{\varepsilon}, v-u^{\varepsilon}\right\rangle+a\left(u^{\varepsilon}, v-u^{\varepsilon}\right)+\left\langle\Phi_{\varepsilon}^{\prime}\left(u^{\varepsilon}\right), v-u^{\varepsilon}\right\rangle \geq\left\langle g, v-u^{\varepsilon}\right\rangle \quad \text { a.e.t } t[0, T] \\
\forall v \in K, \text { and } u^{\varepsilon}(0)=u_{b} .
\end{array}
$$

Let us show first that for all $\mu \in[0,1] u_{4}^{\varepsilon}(\mu) \leq u_{3}^{\varepsilon}(\mu)$, then that $u_{3}^{\varepsilon}(\mu) \rightarrow u_{3}(\mu)$ and $u_{4}^{\varepsilon}(\mu) \rightarrow$ $u_{4}(\mu)$ strongly in $L^{2}(0, T ; H)$ when $\varepsilon \rightarrow 0$. Indeed for all $\mu \in[0,1]$, let consider $U_{\varepsilon}(\mu)=$ $u_{4}^{\varepsilon}(\mu)-u_{3}^{\varepsilon}(\mu)$ thus $u_{4}^{\varepsilon}(\mu)(t)-U_{\varepsilon}^{+}(\mu)(t)$ is in $K$. So we can take $v=u_{4}^{\varepsilon}(\mu)(t)-U_{\varepsilon}^{+}(\mu)(t)$ in (2.10) where $u^{\varepsilon}=u_{4}^{\varepsilon}(\mu)$ and $g=g_{3}(\mu)=\mu\left(g_{1}-g_{2}\right)+g_{2}$. We also can take $v=u_{1}^{\varepsilon}(t)+U_{\varepsilon}^{+}(\mu)(t)$ in (2.10) where $u^{\varepsilon}=u_{1}^{\varepsilon}$ and $g=g_{1}$, and we multiply the two sides of the obtained inequality by $\mu$ then we take $v=u_{2}^{\varepsilon}+U_{\varepsilon}^{+}(\mu)$ in (2.10) where $u^{\varepsilon}=u_{2}^{\varepsilon}$ and $g=g_{2}$ and we multiply the two sides of the obtained inequality by $(1-\mu)$. By adding the three obtained inequalities we get a.e. $t \in] 0, T[$,

$$
\frac{1}{2} \frac{\partial}{\partial t}\left(\left\|U_{\varepsilon}^{+}(\mu)\right\|_{H}^{2}\right)+\lambda\left\|U_{\varepsilon}^{+}(\mu)\right\|_{V}^{2} \leq\left\langle\mu \Phi_{\varepsilon}^{\prime}\left(u_{1}^{\varepsilon}\right)+(1-\mu) \Phi_{\varepsilon}^{\prime}\left(u_{2}^{\varepsilon}\right)-\Phi_{\varepsilon}^{\prime}\left(u_{4}^{\varepsilon}(\mu)\right), U_{\varepsilon}^{+}(\mu)\right\rangle,
$$

hence as $U_{\varepsilon}^{+}(\mu)(0)=0$, by integration from $t=0$ to $t=T$ we obtain a.e. $\left.t \in\right] 0, T[$

$$
\begin{gathered}
\frac{1}{2}\left\|U_{\varepsilon}^{+}(\mu)(T)\right\|_{H}^{2}+\lambda \int_{0}^{T}\left\|U_{\varepsilon}^{+}(\mu)(t)\right\|_{V}^{2} d t \leq \\
\leq \int_{0}^{T}\left\langle\mu \Phi_{\varepsilon}^{\prime}\left(u_{1}^{\varepsilon}(t)\right)+(1-\mu) \Phi_{\varepsilon}^{\prime}\left(u_{2}^{\varepsilon}(t)\right)-\Phi_{\varepsilon}^{\prime}\left(u_{4}^{\varepsilon}(\mu)(t)\right), U_{\varepsilon}^{+}(\mu)(t)\right\rangle d t .
\end{gathered}
$$

As

$$
\begin{aligned}
& <\mu \Phi_{\varepsilon}^{\prime}\left(u_{1}^{\varepsilon}\right)+(1-\mu) \Phi_{\varepsilon}^{\prime}\left(u_{2}^{\varepsilon}\right)-\Phi_{\varepsilon}^{\prime}\left(u_{4}^{\varepsilon}(\mu)\right), U_{\varepsilon}^{+}(\mu)>= \\
& =\int_{\Gamma_{2}^{\prime}} \frac{q \mu u_{1}^{\varepsilon} U_{\varepsilon}^{+}(\mu)}{\sqrt{\varepsilon^{2}+\left|u_{1}^{\varepsilon}\right|^{2}}} d s+\int_{\Gamma_{2}^{\prime}} \frac{q(1-\mu) u_{2}^{\varepsilon} U_{\varepsilon}^{+}(\mu)}{\sqrt{\varepsilon^{2}+\left|u_{2}^{\varepsilon}\right|^{2}}} d s-\int_{\Gamma_{2}^{\prime}} \frac{q u_{4}^{\varepsilon}(\mu) U_{\varepsilon}^{+}(\mu)}{\sqrt{\varepsilon^{2}+\left|u_{4}^{\varepsilon}\right|^{2}}} d s
\end{aligned}
$$

where $\Gamma_{2}^{\prime}=\Gamma_{2} \cap\left\{u_{4}^{\varepsilon}(\mu)>u_{3}^{\varepsilon}(\mu)\right\}$. The function $x \mapsto \psi(x)=\frac{x}{\sqrt{\varepsilon^{2}+x^{2}}}$ for $x \in \mathbb{R}$ is increasing

$$
\begin{aligned}
\left(\psi^{\prime}(x)=\right. & \left.\varepsilon^{2}\left(\varepsilon^{2}+x^{2}\right)^{\frac{-3}{2}}>0\right) \text { so } \\
& \int_{\Gamma_{2}^{\prime}} \frac{q \mu u_{1}^{\varepsilon} U_{\varepsilon}^{+}(\mu)}{\sqrt{\varepsilon^{2}+\left\|u_{1}^{\varepsilon}\right\|_{\mathbb{R}^{N}}^{2}}} d s+\int_{\Gamma_{2}^{\prime}} \frac{q(1-\mu) u_{2}^{\varepsilon} U_{\varepsilon}^{+}(\mu)}{\sqrt{\varepsilon^{2}+\left|u_{2}^{\varepsilon}\right|^{2}}} d s-\int_{\Gamma_{2}^{\prime}} \frac{q u_{4}^{\varepsilon}(\mu) U_{\varepsilon}^{+}(\mu)}{\sqrt{\varepsilon^{2}+\left|u_{4}^{\varepsilon}\right|^{2}}} d s \\
\leq & \int_{\Gamma_{2}^{\prime}} \frac{q \mu u_{1}^{\varepsilon} U_{\varepsilon}^{+}(\mu)}{\sqrt{\varepsilon^{2}+\left|u_{1}^{\varepsilon}\right|^{2}}} d s+\int_{\Gamma_{2}^{\prime}} \frac{q(1-\mu) u_{2}^{\varepsilon} U_{\varepsilon}^{+}(\mu)}{\sqrt{\varepsilon^{2}+\left|u_{2}^{\varepsilon}\right|^{2}}} d s-\int_{\Gamma_{2}^{\prime}} \frac{q u_{3}^{\varepsilon}(\mu) U_{\varepsilon}^{+}(\mu)}{\sqrt{\varepsilon^{2}+\left|u_{3}^{\varepsilon}\right|^{2}}} d s .
\end{aligned}
$$


Moreover the function $\psi$ is concave on $\mathbb{R}^{+} \backslash\{0\}\left(\psi^{\prime \prime}(x)=-3 \varepsilon^{2} x\left(\varepsilon^{2}+x^{2}\right)^{\frac{-5}{2}}<0\right)$ thus

$$
\frac{1}{2}\left\|U^{+}(\mu)(T)\right\|_{H}^{2}+\lambda \int_{0}^{T}\left\|U^{+}(\mu)(t)\right\|_{V}^{2} d t \leq 0 .
$$

As $U_{\varepsilon}^{+}(\mu)=0$ on $\left\{\Gamma_{2} \times[0, T]\right\} \cap\left\{u_{4}^{\varepsilon}(\mu) \leq u_{3}^{\varepsilon}(\mu)\right\}$ so

$$
u_{4}^{\varepsilon}(\mu) \leq u_{3}^{\varepsilon}(\mu) \quad \forall \mu \in[0,1] .
$$

Now we must prove that $u_{3}^{\varepsilon}(\mu) \rightarrow u_{3}(\mu)$ and $u_{4}^{\varepsilon}(\mu) \rightarrow u_{4}(\mu)$ strongly in $L^{2}(0, T ; H)$ when $\varepsilon \rightarrow 0$. Taking in (2.10) $v=u_{b} \in K$ with $u^{\varepsilon}=u_{i}^{\varepsilon}(i=1,2)$, we deduce that

$$
\begin{aligned}
\left\langle\dot{u}_{i}^{\varepsilon}, u_{i}^{\varepsilon}-u_{b}\right\rangle+a\left(u_{i}^{\varepsilon}-u_{b}, u_{i}^{\varepsilon}-u_{b}\right)+ & \left\langle\Phi_{\varepsilon}^{\prime}\left(u_{i}^{\varepsilon}\right), u_{i}^{\varepsilon}\right\rangle \leq a\left(u_{b}, u_{b}-u_{i}^{\varepsilon}\right) \\
& +\left\langle\Phi_{\varepsilon}^{\prime}\left(u_{i}^{\varepsilon}\right), u_{b}\right\rangle-\left\langle g_{i}, u_{b}-u_{i}^{\varepsilon}\right\rangle .
\end{aligned}
$$

As

$$
\left\langle\Phi_{\varepsilon}^{\prime}\left(u_{i}^{\varepsilon}\right), u_{i}^{\varepsilon}\right\rangle \geq 0 \quad \text { and } \quad\left|\left\langle\Phi_{\varepsilon}^{\prime}\left(u_{i}^{\varepsilon}\right), u_{b}\right\rangle\right| \leq \int_{\Gamma_{2}} q\left|u_{b}\right| d s
$$

we deduce, using the Cauchy-Schwartz inequality, that $\left\|u_{i}^{\varepsilon}\right\|_{L^{2}(0, T ; V)}$ so also $\left\|u_{3}^{\varepsilon}(\mu)\right\|_{L^{2}(0, T ; V)}$ are bounded independently from $\varepsilon$. By Theorem 2.2 we get

$$
\begin{array}{r}
\frac{1}{2}\left\|u_{3}^{\varepsilon}(\mu)-u_{4}^{\varepsilon}(\mu)\right\|_{L^{\infty}(0, T ; H)}+\lambda\left\|u_{3}^{\varepsilon}(\mu)-u_{4}^{\varepsilon}(\mu)\right\|_{L^{2}(0, T ; V)} \leq \mu(1-\mu)\left(\mathcal{A}^{\varepsilon}\left(T, g_{1}\right)+\mathcal{B}^{\varepsilon}\left(T, g_{2}\right)\right) \\
\leq \mu(1-\mu) \frac{1}{2}\left(\left\|g_{1}-g_{2}\right\|_{L^{2}(0, T ; H)}^{2}+\left\|u_{1}^{\varepsilon}-u_{2}^{\varepsilon}\right\|_{L^{2}(0, T ; H)}^{2}\right) \quad \forall \mu \in[0,1]
\end{array}
$$

thus $\left\|u_{4}^{\varepsilon}(\mu)\right\|_{L^{2}(0, T ; V)}$ is also bounded independently from $\varepsilon$. So there exists $l_{i} \in V$, for $i=1, \cdots, 4$, such that

$$
u_{i}^{\varepsilon} \rightarrow l_{i} \text { in } L^{2}(0, T ; V) \text { weak, and in } L^{\infty}(0, T ; H) \text { weak star. }
$$

We check now that $l_{i}=u_{i}$. Indeed for $i=1,2$ or 4 and as $\Phi$ is convex functional we have,

$$
\begin{gathered}
\left\langle\dot{u}_{i}^{\varepsilon}, v-u_{i}^{\varepsilon}\right\rangle+a\left(u_{i}^{\varepsilon}, v-u_{i}^{\varepsilon}\right)+\Phi_{\varepsilon}(v)-\Phi_{\varepsilon}\left(u_{i}^{\varepsilon}\right) \geq \\
\left.\left\langle\dot{u}_{i}^{\varepsilon}, v-u_{i}^{\varepsilon}\right\rangle+a\left(u_{i}^{\varepsilon}, v-u_{i}^{\varepsilon}\right)+\left\langle\Phi_{\varepsilon}^{\prime}\left(u_{i}^{\varepsilon}\right), v-u_{i}^{\varepsilon}\right\rangle \geq\left\langle g_{i}, v-u_{i}^{\varepsilon}\right\rangle, \quad \text { a.e.t } \in\right] 0, T[
\end{gathered}
$$

thus

$$
\left.\left\langle\dot{u}_{i}^{\varepsilon}, v-u_{i}^{\varepsilon}\right\rangle+a\left(u_{i}^{\varepsilon}, v-u_{i}^{\varepsilon}\right)+\Phi_{\varepsilon}(v)-\Phi_{\varepsilon}\left(u_{i}^{\varepsilon}\right) \geq\left\langle g_{i}, v-u_{i}^{\varepsilon}\right\rangle, \quad \text { a.e.t } \in\right] 0, T[.
$$

Taking $v=u_{i}^{\varepsilon} \pm \varphi$, in (2.14) we have

$$
\left\langle\dot{u}_{i}^{\varepsilon}, \varphi\right\rangle=-a\left(u_{i}^{\varepsilon}, \varphi\right)+\left\langle g_{i}, \varphi\right\rangle, \quad \forall \varphi \in L^{2}\left(0, T, H_{0}^{1}(\Omega)\right) .
$$

As $H_{0}^{1}(\Omega) \subset V$ with continuous inclusion but not dense, so $V^{\prime}$ (the topological dual of the space $V$ ) is not identifiable with a subset of $H^{-1}(\Omega)$. However, following [28] we can use the Hahn-Banach Theorem in order to extend any element in $H^{-1}(\Omega)$ to an element of $V^{\prime}$ preserving its norm. So from (2.13) and $(\underline{2.15})$ we conclude that

$$
\left.\begin{array}{l}
u_{i}^{\varepsilon} \rightarrow l_{i} \text { in } L^{2}(0, T, V) \text { weak, in } L^{\infty}(0, T, H) \text { weak star, } \\
\text { and } \dot{u}_{i}^{\varepsilon} \rightarrow \dot{l}_{i} \text { in } L^{2}\left(0, T, V^{\prime}\right) \text { weak. }
\end{array}\right\}
$$


Then from (2.14), and following ([22, 38]) we can write

$$
\begin{gathered}
\int_{0}^{T}\left\{\left\langle\dot{u}_{i}^{\varepsilon}, v\right\rangle+a\left(u_{i}^{\varepsilon}, v\right)+\Phi_{\varepsilon}(v)-\left\langle g_{i}, v-u_{i}^{\varepsilon}\right\rangle\right\} d t \geq \int_{0}^{T}\left\{\left\langle\dot{u}_{i}^{\varepsilon}, u_{i}^{\varepsilon}\right\rangle+a\left(u_{i}^{\varepsilon}, u_{i}^{\varepsilon}\right)+\Phi_{\varepsilon}\left(u_{i}^{\varepsilon}\right)\right\} d t \\
=\frac{1}{2}\left\|u_{i}^{\varepsilon}(T)\right\|_{H}^{2}-\frac{1}{2}\left\|u_{b}(T)\right\|_{H}^{2}+\int_{0}^{T}\left\{a\left(u_{i}^{\varepsilon}, u_{i}^{\varepsilon}\right)+\Phi_{\varepsilon}\left(u_{i}^{\varepsilon}\right)\right\} d t .
\end{gathered}
$$

Using the property of $\Phi_{\varepsilon}$ we have $\liminf _{\varepsilon \rightarrow 0} \Phi_{\varepsilon}\left(u_{i}^{\varepsilon}\right) \geq \Phi\left(l_{i}\right)$, and (2.16) we obtain

$$
\int_{0}^{T}\left\{\left\langle i_{i}, v\right\rangle+a\left(l_{i}, v\right)+\Phi(v)-\left\langle g_{i}, v-l_{i}\right\rangle\right\} d t \geq \int_{0}^{T}\left\{\left\langle i_{i}, l_{i}\right\rangle+a\left(l_{i}, l_{i}\right)+\Phi\left(l_{i}\right)\right\} d t .
$$

Let $w \in K$ and any $\left.t_{0} \in\right] 0, T\left[\right.$ then we consider the open interval $\left.\mathcal{O}_{j}=\right] t_{0}-\frac{1}{j}, t_{0}+\frac{1}{j}[\subset] 0, T[$ for $j \in \mathbb{N}^{\star}$ sufficiently large we take in (2.17) $v=\left\{\begin{array}{l}w \text { if } t \in \mathcal{O}_{j}, \\ \left.l_{i}(t) \text { if } t \in\right] 0, T\left[\backslash \mathcal{O}_{j}\right.\end{array}\right.$ to get

$$
\int_{\mathcal{O}_{j}}\left\{\left\langle i_{i}, w-l_{i}\right\rangle+a\left(l_{i}, w-l_{i}\right)+\Phi(w)-\Phi\left(l_{i}\right)\right\} d t \geq \int_{\mathcal{O}_{j}}\left\langle g_{i}, w-l_{i}\right\rangle d t
$$

We use now the Lebesgues Theorem to obtain, when $j \rightarrow+\infty$

$$
\left.\left\langle i_{i}, w-l_{i}\right\rangle+a\left(l_{i}, w-l_{i}\right)+\Phi(w)-\Phi\left(l_{i}\right) \geq\left\langle g_{i}, w-l_{i}\right\rangle, \quad \text { a.e. } t \in\right] 0, T[.
$$

So by the uniqueness of the solution of the parabolic variational inequality of second kind (1.1), we deduce that $l_{i}=u_{i}$.

To finish the proof we check the strong convergence of $u_{i}^{\varepsilon}$ to $u_{i}$. Indeed for $i=1,2$ or 4 taking $v=u_{i}(t)$ in (1.1) where $u=u_{i}^{\varepsilon}$ then $v=u_{i}^{\varepsilon}(t)$ in (1.1) where $u=u_{i}$, then by addition, and integration over the time interval $[0, T]$ we obtain

$$
\begin{array}{r}
\frac{1}{2}\left\|u_{i}(T)-u_{i}^{\varepsilon}(T)\right\|_{H}^{2}+\int_{0}^{T} a\left(u_{i}(t)-u_{i}^{\varepsilon}(t), u_{i}(t)-u_{i}^{\varepsilon}(t)\right) d t \\
\leq \int_{0}^{T} \Phi_{\varepsilon}\left(u_{i}(t)\right)-\Phi\left(u_{i}(t)\right)+\Phi\left(u_{i}^{\varepsilon}(t)\right)-\Phi_{\varepsilon}\left(u_{i}^{\varepsilon}(t)\right) d t
\end{array}
$$

as

$$
\Phi_{\varepsilon}(v)-\Phi(v)=\int_{\Gamma_{2}} q\left(\sqrt{\varepsilon^{2}+|v|^{2}}-|v|\right) d s \leq \varepsilon \sqrt{\left|\Gamma_{2}\right|}\|q\|_{L^{2}\left(\Gamma_{2}\right)},
$$

so from $(2.20)$

$$
\frac{1}{2}\left\|u_{i}-u_{i}^{\varepsilon}\right\|_{L^{\infty}(0, T, H)}^{2}+\int_{0}^{T} a\left(u_{i}(t)-u_{i}^{\varepsilon}(t), u_{i}(t)-u_{i}^{\varepsilon}(t)\right) d t \leq 2 T \varepsilon \sqrt{\left|\Gamma_{2}\right|}\|q\|_{L^{2}\left(\Gamma_{2}\right)}
$$

thus

$$
u_{i}^{\varepsilon} \rightarrow u_{i} \text { strongly in } L^{2}(0, T ; V) \cap L^{\infty}(0, T ; H) \text { for } i=1,2,4
$$

then also

$$
u_{3}^{\varepsilon}(\mu)=\mu u_{1}^{\varepsilon}+(1-\mu) u_{2}^{\varepsilon} \rightarrow u_{3} \text { strongly in } L^{2}(0, T ; V) \cap L^{\infty}(0, T ; H) .
$$

from (2.12), (2.21) and (2.22) we get (2.8). As the proof is given for any two control $g=g_{1}$ and $g=g_{2}$ in $L^{2}(0, T, H)$, but for the same $q, h, b$ and the same initial condition (1.2), so we get also (2.9). 


\subsection{Dependency of the solutions on the data}

Note that this Subsection is not needed in the last Section. We just would like to establish three propositions which allow us to deduce some additional and interesting properties on the solutions of the variational problems $P$ and $P_{h}$.

Proposition 2.6. Let $u_{g_{n}}$, $u_{g}$ be two solutions of Problem $P$, with $g=g_{n}$ and $g=g$ respectively. Assume that $g_{n} \rightarrow g$ in $L^{2}(0, T, H)$ (weak), we get

$$
\begin{gathered}
u_{g_{n}} \rightarrow u_{g} \quad \text { in } \quad L^{2}(0, T, V) \cap L^{\infty}(0, T, H) \quad \text { (strong) } \\
\dot{u}_{g_{n}} \rightarrow \dot{u}_{g} \quad \text { in } \quad L^{2}\left(0, T, V^{\prime}\right) \quad \text { (strong). }
\end{gathered}
$$

Moreover

$$
\begin{gathered}
g_{1} \geq g_{2} \quad \text { in } \quad \Omega \times[0, T] \quad \text { then } \quad u_{g_{1}} \geq u_{g_{2}} \quad \text { in } \quad \Omega \times[0, T] . \\
u_{\min \left(g_{1}, g_{2}\right)} \leq u_{4}(\mu) \leq u_{\max \left(g_{1}, g_{2}\right)}, \quad \forall \mu \in[0,1] .
\end{gathered}
$$

Let $u_{g_{1} h}, u_{g_{2} h}$ be two solutions of Problem $P_{h}$, with $g=g_{1}$ and $g=g_{2}$ respectively for all $h>0$, we get

$$
\begin{gathered}
g_{1} \geq g_{2} \quad \text { in } \quad \Omega \times[0, T] \quad \text { then } \quad u_{g_{1} h} \geq u_{g_{2} h} \quad \text { in } \quad \Omega \times[0, T] . \\
u_{\min \left(g_{1}, g_{2}\right) h} \leq u_{h 4}(\mu) \leq u_{\max \left(g_{1}, g_{2}\right) h} \quad \forall \mu \in[0,1] .
\end{gathered}
$$

Proof. Let $g_{n} \rightarrow g$ in $L^{2}(0, T, H), u_{g_{n}}$ and $u_{g}$ be in $L^{2}(0, T, K)$ such that

$$
\begin{array}{r}
\left\langle\dot{u}_{g_{n}}, v-u_{g_{n}}\right\rangle+a\left(u_{g_{n}}, v-u_{g_{n}}\right)+\Phi(v)-\Phi\left(u_{g_{n}}\right) \geq\left(g_{n}, v-u_{g_{n}}\right) \\
\forall v \in K, \quad \text { a.e.t } \in] 0, T[.
\end{array}
$$

Remark also that $V_{2}=\left\{v \in V: \quad v_{\Gamma_{2}}=0\right\} \subset V$ with continuous inclusion but not dense, so $V^{\prime}$ is not identifiable with a subset of $V_{2}^{\prime}$. However, following again [28] we can use the Hahn-Banach Theorem in order to extend any element in $V_{2}^{\prime}$ to an element of $V^{\prime}$ preserving its norm. So with the same arguments as in (2.14)- (2.19), we conclude that there exists $\eta$ such that (eventually for a subsequence)

$$
\left.\begin{array}{l}
u_{g_{n}} \rightarrow \eta \text { in } L^{2}(0, T, V) \text { weak, in } L^{\infty}(0, T, H) \text { weak star, } \\
\text { and } \dot{u}_{g_{n}} \rightarrow \dot{\eta} \text { in } L^{2}\left(0, T, V^{\prime}\right) \text { weak }
\end{array}\right\}
$$

Using (2.30) and taking $n \rightarrow+\infty$ in (2.29), we get

$$
\left.\langle\dot{\eta}, v-\eta\rangle+a(\eta, v-\eta)+\Phi(v)-\Phi\left(u_{\eta}\right) \geq(g, v-\eta), \quad \forall v \in K, \quad \text { a.e.t } \in\right] 0, T[,
$$

by the uniqueness of the solution of (1.1) we obtain that $\eta=u_{g}$. Taking now $v=u_{g}(t)$ in (2.29) and $v=u_{g_{n}}(t)$ in (2.31), we get by addition and integration over $[0, T]$ we obtain

$$
\frac{1}{2}\left\|u_{g_{n}}(T)-u_{g}(T)\right\|_{H}^{2}+\lambda\left\|u_{g_{n}}-u_{g}\right\|_{L^{2}(0, T, V)}^{2} \leq \int_{0}^{T}\left(g_{n}(t)-g(t), u_{g_{n}}(t)-u_{g}(t)\right) d t,
$$

so from the above inequality and (2.30) we deduce (2.23). To prove (2.25) we take first $v=u_{1}(t)+\left(u_{1}(t)-u_{2}(t)\right)^{-}$(which is in $\left.K\right)$ in (1.1) where $u=u_{1}$ and $g=g_{1}$, then taking $v=u_{2}(t)-\left(u_{1}(t)-u_{2}(t)\right)^{-}$(which also is in $\left.K\right)$ in (1.1) where $u=u_{2}$ and $g=g_{2}$, we get

$$
\frac{1}{2}\left\|\left(u_{1}(T)-u_{2}(T)\right)^{-}\right\|_{H}^{2}+\lambda\left\|\left(u_{1}-u_{2}\right)^{-}\right\|_{L^{2}(0, T, V)}^{2} \leq \int_{0}^{T}\left(g_{2}(t)-g_{1}(t),\left(u_{1}(t)-u_{2}(t)\right)^{-}\right) d t
$$


as

$$
\Phi\left(u_{1}\right)-\Phi\left(u_{1}+\left(u_{1}-u_{2}\right)^{-}\right)+\Phi\left(u_{2}\right)-\Phi\left(u_{2}-\left(u_{1}-u_{2}\right)^{-}\right)=0 .
$$

So if $g_{2}-g_{1} \leq 0$ in $\Omega \times[0, T]$ then $\left\|\left(u_{1}-u_{2}\right)^{-}\right\|_{L^{2}(0, T, V)}=0$, and as $\left(u_{1}-u_{2}\right)^{-}=0$ on $\left.\Gamma_{1} \times\right] 0, T$ [ we have by the Poincaré inequality that $u_{1}-u_{2} \geq 0$ in $\Omega \times[0, T]$. Then (2.26) follows from (2.25) because

$$
\min \left\{g_{1}, g_{2}\right\} \leq \mu g_{1}+(1-\mu) g_{2} \leq \max \left\{g_{1}, g_{2}\right\} \quad \forall \mu \in[0, T] .
$$

Similarly taking $v=u_{g_{1} h}(t)+\left(u_{g_{1} h}(t)-u_{g_{2} h}(t)\right)^{-}$(which is in $V$ ) in (2.1) where $u=u_{g_{1} h}$ and $g=g_{1} h$, then taking $v=u_{g_{2} h}(t)-\left(u_{g_{1} h}(t)-u_{g_{2} h}(t)\right)^{-}$(which also is in $V$ ) in (2.1) where $u=u_{g_{2}}$ and $g=g_{2} h$, we get

$$
\begin{array}{r}
\frac{1}{2}\left\|\left(u_{g_{1} h}(T)-u_{g_{2} h}(T)\right)^{-}\right\|_{H}^{2}+\lambda\left\|\left(u_{g_{1} h}-u_{g_{2} h}\right)^{-}\right\|_{L^{2}(0, T, V)}^{2}+h\left\|\left(u_{g_{1} h}-u_{g_{2} h}\right)^{-}\right\|_{L^{2}\left(0, T, L^{2}\left(\Gamma_{1}\right)\right)}^{2} \\
\leq \int_{0}^{T}\left(g_{2}(t)-g_{1}(t),\left(u_{1}(t)-u_{2}(t)\right)^{-}\right) d t
\end{array}
$$

so we get also (2.27), then (2.28) follows.

The following propositions 2.7 and 2.8 are to give, with some assumptions, a first information that the sequence $\left(u_{g_{h}}\right)_{h>0}$ is increasing and bounded, therefore it is convergent in some sense. Remark from (2.4) that $u_{g_{h}} \geq 0$ although $g<0$, provided to take the parameter $h$ sufficiently large.

Proposition 2.7. Assume that $h>0$ and is sufficiently large, $b$ is a positive constant, $q \geq 0$ on $\Gamma_{2} \times[0, T]$, then we have

$$
g \leq 0 \text { in } \Omega \times[0, T] \Longrightarrow 0 \leq u_{g_{h}} \leq b \text { in } \Omega \cup \Gamma_{1} \times[0, T],
$$

Proof. Taking in (2.1) $u=u_{g_{h}}(t)$ and $v=u_{g_{h}}(t)-\left(u_{g_{h}}(t)-b\right)^{+}$, we get

$$
\begin{aligned}
\left\langle\dot{u}_{g_{h}},\left(u_{g_{h}}-b\right)^{+}\right\rangle & +a_{h}\left(u_{g_{h}},\left(u_{g_{h}}-b\right)^{+}\right)-\Phi\left(u_{g_{h}}-\left(u_{g_{h}}-b\right)^{+}\right)+\Phi\left(u_{g_{h}}\right) \\
\leq & \left.\left(g,\left(u_{g_{h}}-b\right)^{+}\right)+h \int_{\Gamma_{1}} b\left(u_{g_{h}}-b\right)^{+} d s, \quad \text { a.e.t } \in\right] 0, T[
\end{aligned}
$$

as $b$ is constant we have $a\left(b,\left(u_{g_{h}}(t)-b\right)^{+}\right)=0$ so a.e. $\left.t \in\right] 0, T[$

$$
\begin{array}{r}
\frac{1}{2} \frac{\partial}{\partial t}\left(\left\|\left(u_{g_{h}}(t)-b\right)^{+}\right\|_{H}^{2}\right)+a\left(\left(u_{g_{h}}-b\right)^{+},\left(u_{g_{h}}-b\right)^{+}\right)+h \int_{\Gamma_{1}} u_{g_{h}}\left(u_{g_{h}}-b\right)^{+} d s \\
\leq\left(g,\left(u_{g_{h}}-b\right)^{+}\right)+h \int_{\Gamma_{1}} b\left(u_{g_{h}}-b\right)^{+} d s+\Phi\left(u_{g_{h}}-\left(u_{g_{h}}-b\right)^{+}\right)-\Phi\left(u_{g_{h}}\right),
\end{array}
$$

as $u_{g_{h}}(0)=b$ and

$$
\Phi\left(u_{g_{h}}-\left(u_{g_{h}}-b\right)^{+}\right)-\Phi\left(u_{g_{h}}\right)=\int_{\Gamma_{2}} q\left(\left|u_{g_{h}}-\left(u_{g_{h}}-b\right)^{+}\right|-\left|u_{g_{h}}\right|\right) d s \leq 0,
$$

so

$$
\begin{array}{r}
\frac{1}{2}\left\|\left(u_{g_{h}}(T)-b\right)^{+}\right\|_{H}^{2}+\int_{0}^{T} a_{h}\left(\left(u_{g_{h}}(t)-b\right)^{+},\left(u_{g_{h}}(t)-b\right)^{+}\right) d t \leq \\
\leq \int_{0}^{T}\left(g(t),\left(u_{g_{h}}(t)-b\right)^{+}\right) d t \leq 0,
\end{array}
$$

thus (2.32) holds. 
Proposition 2.8. Assume that $h>0$ and is sufficiently large. Let $g, g_{1}, g_{2}$ in $L^{2}(0, T, H)$, $q \in L^{2}\left(0, T, L^{2}\left(\Gamma_{2}\right)\right)$ and $b$ is a positive constant, we have

$$
\begin{gathered}
g_{2} \leq g_{1} \leq 0 \text { in } \Omega \times[0, T] \quad \text { and } \quad h_{2} \leq h_{1} \Longrightarrow 0 \leq u_{g_{2} h_{2}} \leq u_{g_{1} h_{1}} \text { in } \Omega \times[0, T], \\
g \leq 0 \text { in } \Omega \times[0, T] \Longrightarrow 0 \leq u_{g_{h}} \leq u_{g} \text { in } \Omega \times[0, T], \quad \forall h>0 . \\
h_{2} \leq h_{1} \Longrightarrow\left\|u_{g_{h_{2}}}-u_{g_{h_{1}}}\right\|_{L^{2}(0, T, V)} \leq \frac{\left\|\gamma_{0}\right\|}{\lambda_{1} \min \left(1, h_{2}\right)}\left\|b-u_{g_{h_{1}}}\right\|_{L^{2}\left(0, T, \mathbf{L}^{2}\left(\Gamma_{1}\right)\right)}\left(h_{1}-h_{2}\right)
\end{gathered}
$$

Proof. To check (2.33) we take first $v=u_{g_{1} h_{1}}(t)+\left(u_{g_{2} h_{2}}(t)-u_{g_{1} h_{1}}(t)\right)^{+}$, for $t \in[0, T]$, in (2.1) where $u=u_{g_{1} h_{1}}, g=g_{1} h_{1}$ and $h=h_{1}$, then taking $v=u_{g_{2} h_{2}}(t)-\left(u_{g_{2} h_{2}}(t)-u_{g_{1} h_{1}}(t)\right)^{+}$ in (2.1) where $u=u_{g_{2} h_{2}}, g=g_{2} h_{2}$ and $h=h_{2}$, adding the two obtained inequalities, as

$$
\left.\Phi\left(u_{g_{1} h_{1}}+\left(u_{g_{2} h_{2}}-u_{g_{1} h_{1}}\right)^{+}\right)-\Phi\left(u_{g_{1} h_{1}}\right)+\Phi\left(u_{g_{2} h_{2}}-\left(u_{g_{2} h_{2}}-u_{g_{1} h_{1}}\right)^{+}\right)\right)-\Phi\left(u_{g_{2} h_{2}}\right)=0
$$

we get

$$
\begin{array}{r}
-\frac{1}{2} \frac{\partial}{\partial t}\left(\left\|\left(u_{g_{2} h_{2}}-u_{g_{1} h_{1}}\right)^{+}\right\|_{H}^{2}\right)-a\left(u_{g_{2} h_{2}}-u_{g_{1} h_{1}},\left(u_{g_{2} h_{2}}-u_{g_{1} h_{1}}\right)^{+}\right) \\
+\int_{\Gamma_{1}}\left(h_{1} u_{g_{1} h_{1}}-h_{2} u_{g_{2} h_{2}}\right)\left(u_{g_{2} h_{2}}-u_{g_{1} h_{1}}\right)^{+} d s \geq\left(g_{1}-g_{2},\left(u_{g_{2} h_{2}}-u_{g_{1} h_{1}}\right)^{+}\right) \\
\left.+\left(h_{1}-h_{2}\right) \int_{\Gamma_{1}} b\left(u_{g_{2} h_{2}}-u_{g_{1} h_{1}}\right)^{+} d s, \quad \text { a.e.t } \in\right] 0, T[
\end{array}
$$

so by integration on $] 0, T[$, we deduce

$$
\begin{gathered}
\frac{1}{2}\left\|\left(u_{g_{2} h_{2}}(T)-u_{g_{1} h_{1}}(T)\right)^{+}\right\|_{H}^{2}+\int_{0}^{T} a_{h_{2}}\left(\left(u_{g_{2} h_{2}}-u_{g_{1} h_{1}}\right)^{+},\left(u_{g_{2} h_{2}}-u_{g_{1} h_{1}}(t)\right)^{+}\right) d t \leq \\
\int_{0}^{T}\left(g_{2}-g_{1},\left(u_{g_{2} h_{2}}(t)-u_{g_{1} h_{1}}\right)^{+}\right) d t+\left(h_{1}-h_{2}\right) \int_{0}^{T} \int_{\Gamma_{1}}\left(u_{g_{1} h_{1}}-b\right)\left(u_{g_{2} h_{2}}-u_{g_{1} h_{1}}\right)^{+} d s d t,
\end{gathered}
$$

and from (2.32) we get (2.33). To check (2.34), let $W=u_{g_{h}}(t)-u_{g}(t)$, and choose, in (2.1), $v=u_{g_{h}}(t)-W^{+}(t)$, so a.e. $\left.t \in\right] 0, T[$

$$
\left\langle\dot{u}_{g_{h}}, W^{+}\right\rangle+a_{h}\left(u_{g_{h}}, W^{+}\right) \leq+\Phi\left(u_{g_{h}}-W^{+}\right)-\Phi\left(u_{g_{h}}\right)+\left(g, W^{+}\right)+h \int_{\Gamma_{1}} b W^{+} d s
$$

as $u_{g}=b$ on $\Gamma_{1} \times[0, T]$ we obtain a.e. $\left.t \in\right] 0, T[$

$$
\left\langle\dot{u}_{g_{h}}, W^{+}\right\rangle+a\left(u_{g_{h}}, W^{+}\right)+h \int_{\Gamma_{1}}\left|W^{+}\right|^{2} d s \leq\left(g, W^{+}\right)+\Phi\left(u_{g_{h}}-W^{+}\right)-\Phi\left(u_{g_{h}}\right) .
$$

Then we choose, in (1.1), $v=u_{g}(t)+W^{+}(t)$, which is in $K$ because from (2.32) we have $W^{+}=0$ on $\Gamma_{1} \times[0, T]$, so

$$
\left.\left\langle\dot{u}_{g}, W^{+}(t)\right\rangle+a\left(u_{g}, W^{+}\right) \geq\left(g, W^{+}\right)-\Phi\left(u_{g}+W^{+}\right)+\Phi\left(u_{g}\right), \quad \text { a.e. } t \in\right] 0, T[.
$$

So from (2.36) and (2.37) we deduce that

$$
\begin{aligned}
& \frac{1}{2}\left\|W^{+}(T)\right\|_{H}^{2}+\int_{0}^{T} a\left(W^{+}, W^{+}\right) d t+h \int_{\Gamma_{1}}\left|W^{+}\right|^{2} d s \\
\leq & \Phi\left(u_{g_{h}}-W^{+}\right)-\Phi\left(u_{g_{h}}\right)+\Phi\left(u_{g}+W^{+}\right)-\Phi\left(u_{g}\right)=0 .
\end{aligned}
$$


Then (2.34) holds. To finish the proof we must check (2.35). We choose $v=u_{g_{h_{1}}}(t)$ in (2.1) where $u=u_{g_{h_{2}}}(t)$, then choosing $v=u_{g_{h_{2}}}(t)$ in (2.1) where $u=u_{g_{h_{1}}}(t)$, we get

$$
\begin{array}{r}
-\left\langle\dot{u}_{g_{h_{2}}}-\dot{u}_{g_{h_{1}}}, u_{g_{h_{2}}}-u_{g_{h_{1}}}\right\rangle-a\left(u_{g_{h_{2}}}-u_{g_{h_{1}}}, u_{g_{h_{2}}}-u_{g_{h_{1}}}\right) \\
-h_{2} \int_{\Gamma_{1}} u_{g_{h_{2}}}\left(u_{g_{h_{2}}}-u_{g_{h_{1}}}\right) d s+h_{1} \int_{\Gamma_{1}} u_{g_{h_{1}}}\left(u_{g_{h_{2}}}-u_{g_{h_{1}}}\right) d s \geq \\
\left.-\left(h_{2}-h_{1}\right) \int_{\Gamma_{1}} b\left(u_{g_{h_{2}}}-u_{g_{h_{1}}}\right) d s, \quad \text { a.e. } t \in\right] 0, T[
\end{array}
$$

then

$$
\begin{array}{r}
\frac{1}{2}\left\|u_{g_{h_{2}}}(T)-u_{g_{h_{1}}}(T)\right\|_{H}^{2}+\int_{0}^{T} a_{h_{2}}\left(u_{g_{h_{2}}}-u_{g_{h_{1}}}, u_{g_{h_{2}}}-u_{g_{h_{1}}}\right) d t \\
\leq\left(h_{1}-h_{2}\right) \int_{0}^{T} \int_{\Gamma_{1}}\left(u_{g_{h_{1}}}-b\right)\left(u_{g_{h_{2}}}-u_{g_{h_{1}}}\right) d s d t
\end{array}
$$

So

$$
\begin{aligned}
& \frac{1}{2}\left\|u_{g_{h_{2}}}-u_{g_{h_{1}}}\right\|_{L^{\infty}(0, T, H)}^{2}+\lambda_{1} \min \left\{1, h_{2}\right\}\left\|u_{g_{h_{2}}}-u_{g_{h_{1}}}\right\|_{L^{2}(0, T, V)}^{2} \\
& \quad \leq\left\|\gamma_{0}\right\|\left(h_{1}-h_{2}\right)\left\|b-u_{g_{h_{1}}}\right\|_{L^{2}\left(0, T, \mathbf{L}^{2}\left(\Gamma_{1}\right)\right)}\left\|u_{g_{h_{2}}}-u_{g_{h_{1}}}\right\|_{L^{2}(0, T, V)}
\end{aligned}
$$

where $\gamma_{0}$ is the trace embedding from $V$ to $L^{2}\left(\Gamma_{1}\right)$. Thus (2.35) holds.

\section{Optimal Control problems and convergence for $h \rightarrow+\infty$}

In this section, $b$ is not constant but a given function in $L^{2}(] 0, T\left[\times \Gamma_{1}\right)$. We prove first the existence and uniqueness of the solution for the optimal control problem associated to the parabolic variational inequalities of second kind (1.1), and for the optimal control problem associated also to (2.1), then in Subsection 3.1 we prove (see Lemma 3.2 and Theorem 3.3 ) the convergence of the state $u_{g_{o p_{h}} h}$ and the optimal control $g_{o p_{h}}$, when the coefficient $h$ on $\Gamma_{1}$, goes to infinity.

The existence and uniqueness of the solution to the parabolic variational inequalities of second kind (1.1) and (2.1), with the initial condition (1.2), allow us to consider $g \mapsto u_{g}$ and $g \mapsto u_{g_{h}}$ as functions from $L^{2}(0, T, H)$ to $L^{2}(0, T, V)$, for all $h>0$.

Using the monotony property (2.8) and (2.9), established in Theorem 2.5, we prove in the following that $J$ and $J_{h}$, defined by (1.3) and (1.8), are strictly convex applications on $L^{2}(0, T, H)$, so [27] there exists a unique solution $g_{o p}$ in $L^{2}(0, T, H)$ of the Problem (1.9), and there exists also a unique solution $g_{o p_{h}}$ in $L^{2}(0, T, H)$ of Problem (1.7) for all $h>0$.

Theorem 3.1. Assume the same hypotheses of Proposition 2.1. Then $J$ and $J_{h}$, defined by (1.3) and (1.8) respectively, are strictly convex applications on $L^{2}(0, T, H)$, so there exist unique solutions $g_{o p}$ and $g_{o p_{h}}$ in $L^{2}(0, T, H)$ respectively of the Problems (1.9) and (1.7).

Proof. Let $u=u_{g_{i}}$ and $u_{g_{i} h}$ be respectively the solution of the variational inequalities (1.1) and (2.1) with $g=g_{i}$ for $i=1,2$. We have

$$
\left\|u_{3}(\mu)\right\|_{L^{2}(0, T, H)}^{2}=\mu^{2}\left\|u_{g_{1}}\right\|_{L^{2}(0, T, H)}^{2}+(1-\mu)^{2}\left\|u_{g_{2}}\right\|_{L^{2}(0, T, H)}^{2}+2 \mu(1-\mu)\left(u_{g_{1}}, u_{g_{2}}\right)
$$

then the following equalities hold

$$
\begin{array}{r}
\left\|u_{3}(\mu)\right\|_{L^{2}(0, T, H)}^{2}=\mu\left\|u_{g_{1}}\right\|_{L^{2}(0, T, H)}^{2}+(1-\mu)\left\|u_{g_{2}}\right\|_{L^{2}(0, T, H)}^{2} \\
-\mu(1-\mu)\left\|u_{g_{2}}-u_{g_{1}}\right\|_{L^{2}(0, T, H)}^{2},
\end{array}
$$




$$
\begin{array}{r}
\left\|u_{3 h}(\mu)\right\|_{L^{2}(0, T, H)}^{2}=\mu\left\|u_{g_{1} h}\right\|_{L^{2}(0, T, H)}^{2}+(1-\mu)\left\|u_{g_{2} h}\right\|_{L^{2}(0, T, H)}^{2} \\
-\mu(1-\mu)\left\|u_{g_{2} h}-u_{g_{1} h}\right\|_{L^{2}(0, T, H)}^{2} .
\end{array}
$$

Let now $\mu \in[0,1]$ and $g_{1}, g_{2} \in L^{2}(0, T, H)$ so

$$
\begin{array}{r}
\mu J\left(g_{1}\right)+(1-\mu) J\left(g_{2}\right)-J\left(g_{3}(\mu)\right)=\frac{\mu}{2}\left\|u_{g_{1}}\right\|_{L^{2}(0, T, H)}^{2}+\frac{(1-\mu)}{2}\left\|u_{g_{2}}\right\|_{L^{2}(0, T, H)}^{2} \\
-\frac{1}{2}\left\|u_{4}(\mu)\right\|_{L^{2}(0, T, H)}^{2}+\frac{M}{2}\left\{\mu\left\|g_{1}\right\|_{L^{2}(0, T, H)}^{2}+(1-\mu)\left\|g_{2}\right\|_{L^{2}(0, T, H)}^{2}-\left\|g_{3}(\mu)\right\|_{L^{2}(0, T, H)}^{2}\right\}
\end{array}
$$

using (3.1) and $g_{3}(\mu)=\mu g_{1}+(1-\mu) g_{2}$ we obtain

$$
\begin{array}{r}
\mu J\left(g_{1}\right)+(1-\mu) J\left(g_{2}\right)-J\left(g_{3}(\mu)\right)=\frac{1}{2}\left(\left\|u_{3}(\mu)\right\|_{L^{2}(0, T, H)}^{2}-\left\|u_{4}(\mu)\right\|_{L^{2}(0, T, H)}^{2}\right) \\
+\frac{1}{2} \mu(1-\mu)\left\|u_{1}-u_{2}\right\|_{L^{2}(0, T, H)}^{2}+\frac{M}{2} \mu(1-\mu)\left\|g_{1}-g_{2}\right\|_{L^{2}(0, T, H)}^{2},
\end{array}
$$

for all $\mu \in] 0,1\left[\right.$ and for all $g_{1}, g_{2}$ in $L^{2}(0, T, H)$. From Proposition 2.1 we have $u_{4}(\mu) \geq 0$ in $\Omega \times[0, T]$ for all $\mu \in[0,1]$, so using the monotony property (2.8) (Theorem 2.5) and we deduce

$$
\left\|u_{4}(\mu)\right\|_{L^{2}(0, T, H)}^{2} \leq\left\|u_{3}(\mu)\right\|_{L^{2}(0, T, H)}^{2} .
$$

Finally from (3.3) the cost functional $J$ is strictly convex, thus [27 the uniqueness of the optimal control of the problem (1.9) holds.

The uniqueness of the optimal control of the problem (1.7) follows using the analogous inequalities (3.3)-(3.4) for any $h>0$.

\subsection{Convergence when $h \rightarrow+\infty$}

In this last subsection we study the convergence of the state $u_{g_{o p_{h}} h}$ and the optimal control $g_{o p_{h}}$, when the coefficient $h$ on $\Gamma_{1}$, goes to infinity. For a given $g$ in $L^{2}(0, T, H)$ we have first the following estimate which generalizes [36, 37].

Lemma 3.2. Let $u_{g_{h}}$ be the unique solution of the parabolic variational inequality (2.1) and $u_{g}$ the unique solution of the parabolic variational inequality (1.1), then

$$
u_{g_{h}} \rightarrow u_{g} \in L^{2}(0, T, V) \text { strongly as } h \rightarrow+\infty, \quad \forall g \in L^{2}(0, T, H) .
$$

Proof. We take $v=u_{g}(t)$ in (2.1) where $u=u_{g_{h}}$, and recalling that $u_{g}(t)=b$ on $\left.\Gamma_{1} \times\right] 0, T$, taking $u_{g_{h}}(t)-u_{g}(t)=\phi_{h}(t)$ we obtain for $h>1$, a.e. $\left.t \in\right] 0, T[$

$$
\left\langle\dot{\phi}_{h}, \phi_{h}\right\rangle+a_{1}\left(\phi_{h}, \phi_{h}\right)+(h-1) \int_{\Gamma_{1}}\left|\phi_{h}\right|^{2} d s \leq-\left\langle\dot{u}_{g}, \phi_{h}\right\rangle-a\left(u_{g}, \phi_{h}\right)+\left(g, \phi_{h}\right)+\Phi\left(\phi_{h}\right),
$$

so we deduce that

$$
\frac{1}{2}\left\|\phi_{h}\right\|_{L^{\infty}(0, T, H)}^{2}+\left\|\phi_{h}\right\|_{L^{2}(0, T, V)}^{2}+(h-1)\left\|\phi_{h}\right\|_{L^{2}\left(0, T, L^{2}\left(\Gamma_{1}\right)\right)}^{2}
$$

is bounded for all $h>1$, then $\left\|u_{g_{h}}\right\|_{L^{2}(0, T, V)} \leq\left\|\phi_{h}\right\|_{L^{2}(0, T, V)}+\left\|u_{g}\right\|_{L^{2}(0, T, V)}$ is also bounded for all $h>1$. So there exists $\eta \in L^{2}(0, T, V)$ such that $u_{g_{h}} \rightarrow \eta$ weakly in $L^{2}(0, T, V)$ and $u_{g_{h}} \rightarrow b$ strongly on $\Gamma_{1}$ when $h \rightarrow+\infty$ so $\eta(0)=b$. 
Let $\varphi \in L^{2}\left(0, T, V_{2}\right)$ and taking in (2.1) where $u=u_{g_{h}}, v=u_{g_{h}}(t) \pm \varphi(t)$, we obtain

$$
\left.\left\langle\dot{u}_{g_{h}}, \varphi\right\rangle=-a\left(u_{g_{h}}, \varphi\right)+(g, \varphi) \quad \text { a.e.t } \in\right] 0, T[.
$$

As $\left\|u_{g_{h}}\right\|_{L^{2}(0, T, V)}$ is bounded for all $h>1$, we deduce that $\left\|\dot{u}_{g_{h}}\right\|_{L^{2}\left(0, T, V_{2}^{\prime}\right)}$ is also bounded for all $h>1$. Following the proof of Lemma 2.3, we conclude that

$$
\left.\begin{array}{l}
u_{g_{h}} \rightarrow \eta \text { in } L^{2}(0, T, V) \text { weak, and in } L^{\infty}(0, T, H) \text { weak star, } \\
\text { and } \dot{u}_{g_{n}} \rightarrow \dot{\eta} \text { in } L^{2}\left(0, T, V^{\prime}\right) \text { weak. }
\end{array}\right\}
$$

From (2.1) and taking $v \in K$ so $v=b$ on $\Gamma_{1}$, we obtain

$$
\begin{array}{r}
\left\langle\dot{u}_{g_{h}}, v-u_{g_{h}}\right\rangle+a\left(u_{g_{h}}, v-u_{g_{h}}\right)-h \int_{\Gamma_{1}}\left|u_{g_{h}}-b\right|^{2} d s \geq \\
\left.\Phi\left(u_{g_{h}}\right)-\Phi(v)+\left(g, v-u_{g_{h}}\right) \quad \forall v \in K, \quad \text { a.e.t } \in\right] 0, T[
\end{array}
$$

then

$$
\left.\left\langle\dot{u}_{g_{h}}, v-u_{g_{h}}\right\rangle+a\left(u_{g_{h}}, v-u_{g_{h}}\right) \geq \Phi\left(u_{g_{h}}\right)-\Phi(v)+\left(g, v-u_{g_{h}}\right) \quad \forall v \in K \text {, a.e.t } \in\right] 0, T[\text {. }
$$

So with (3.5) and the same arguments as in (2.14) $-(2.19)$, we obtain

$$
\langle\dot{\eta}, v-\eta\rangle+a(\eta, v-\eta)+\Phi(v)-\Phi(\eta) \geq(g, v-\eta) \quad \forall v \in K, \quad \text { a.e. } t \in] 0, T[.
$$

and $\eta(0)=b$. Using the uniqueness of the solution of (1.1)-(1.2) we get that $\eta=u_{g}$.

To prove the strong convergence, we take $v=u_{g}(t)$ in (2.1)

$$
\begin{array}{r}
\left\langle\dot{u}_{g_{h}}, u_{g}-u_{g_{h}}\right\rangle+a_{h}\left(u_{g_{h}}, u_{g}-u_{g_{h}}\right)+\Phi\left(u_{g}\right)-\Phi\left(u_{g_{h}}\right) \geq\left(g, u_{g}-u_{g_{h}}\right) \\
\left.+h \int_{\Gamma_{1}} b\left(u_{g}-u_{g_{h}}\right) d s, \quad \text { a.e. } t \in\right] 0, T[
\end{array}
$$

thus as $u_{g}=b$ on $\left.\Gamma_{1} \times\right] 0, T\left[\right.$, we put $u_{g_{h}}-u_{g}=\phi_{h}$, so a.e. $\left.t \in\right] 0, T[$

$$
\left\langle\dot{\phi}_{h}, \phi_{h}\right\rangle+a\left(\phi_{h}, \phi_{h}\right)+h \int_{\Gamma_{1}}\left|\phi_{h}\right|^{2} d s+\Phi\left(u_{g_{h}}\right)-\Phi\left(u_{g}\right) \leq\left\langle\dot{u}_{g}, \phi_{h}\right\rangle+a\left(u_{g}, \phi_{h}\right)+\left(g, \phi_{h}\right),
$$

SO

$$
\begin{aligned}
& \frac{1}{2}\left\|\phi_{h}\right\|_{L^{\infty}(0, T, H)}^{2}+\lambda_{h}\left\|\phi_{h}\right\|_{L^{2}(0, T, V)}^{2}+\Phi\left(u_{g_{h}}\right)-\Phi\left(u_{g}\right) \leq-\int_{0}^{T}\left\langle\dot{u}_{g}(t), \phi_{h}(t)\right\rangle d t \\
& -\int_{0}^{T} a\left(u_{g}(t), \phi_{h}(t) d t+\int_{0}^{T}\left(g(t), \phi_{h}(t) d t,\right.\right.
\end{aligned}
$$

using the weak semi-continuity of $\Phi$ and the weak convergence (2.30) the right side of the just above inequality tends to zero when $h \rightarrow+\infty$, then we deduce the strong convergence of $\phi_{h}=u_{g_{h}}-u_{g}$ to 0 in $L^{2}(0, T, V) \cap L^{\infty}(0, T, H)$, for all $g \in L^{2}(0, T, H)$. This ends the proof.

We give now, without need to use the notion of adjoint states [27, the convergence result which generalizes the result obtained in [28] for a parabolic variational equations (see also [3, 6, 23, 24]). 
Theorem 3.3. Let $u_{g_{o p_{h}} h}, g_{o p_{h}}$ and $u_{g_{o p}}, g_{o p}$ be respectively the states and the optimal control defined in the problems (1.9) and (1.7). Then

$$
\begin{aligned}
\lim _{h \rightarrow+\infty}\left\|u_{g_{o p_{h}}}-u_{g_{o p}}\right\|_{L^{2}(0, T, V)} & =\lim _{h \rightarrow+\infty}\left\|u_{g_{o p_{h}}}-u_{g_{o p}}\right\|_{L^{\infty}(0, T, H)}, \\
& =\lim _{h \rightarrow+\infty}\left\|u_{g_{o p_{h}}}-u_{g_{o p}}\right\|_{L^{2}\left(0, T, L^{2}\left(\Gamma_{1}\right)\right)}=0, \\
\lim _{h \rightarrow+\infty} \| g_{o p_{h}} & -g_{o p} \|_{L^{2}(0, T, H)}=0 .
\end{aligned}
$$

Proof. We have first

$$
J_{h}\left(g_{o p_{h}}\right)=\frac{1}{2}\left\|u_{g_{o p_{h} h}}\right\|_{L^{2}(0, T, H)}^{2}+\frac{M}{2}\left\|g_{o p_{h}}\right\|_{L^{2}(0, T, H)}^{2} \leq \frac{1}{2}\left\|u_{g_{h}}\right\|_{L^{2}(0, T, H)}^{2}+\frac{M}{2}\|g\|_{L^{2}(0, T, H)}^{2},
$$

for all $g \in L^{2}(0, T, H)$, then for $g=0 \in L^{2}(0, T, H)$ we obtain that

$$
J_{h}\left(g_{o p_{h}}\right)=\frac{1}{2}\left\|u_{g_{o p_{h} h}}\right\|_{L^{2}(0, T, H)}^{2}+\frac{M}{2}\left\|g_{o p_{h}}\right\|_{L^{2}(0, T, H)}^{2} \leq \frac{1}{2}\left\|u_{0_{h}}\right\|_{L^{2}(0, T, H)}^{2}
$$

where $u_{0_{h}} \in L^{2}(0, T, V)$ is the solution of the following parabolic variational inequality

$$
\left.\left\langle\dot{u}_{0_{h}}, v-u_{0_{h}}\right\rangle+a_{h}\left(u_{0_{h}}, v-u_{0_{h}}\right)+\Phi(v)-\Phi\left(u_{0_{h}}\right) \geq h \int_{\Gamma_{1}} b\left(v-u_{0_{h}}\right) d s, \quad \text { a.e.t } \in\right] 0, T[
$$

for all $v \in V$ and $u_{0_{h}}(0)=u_{b}$. Taking $v=u_{b} \in K$ we get that $\left\|u_{0_{h}}-u_{b}\right\|_{L^{2}(0, T, V)}$ is bounded independently of $h$, then $\left\|u_{0_{h}}\right\|_{L^{2}(0, T, H)}$ is bounded independently of $h$. So we deduce with (3.9) that $\left\|u_{g_{o p_{h}} h}\right\|_{L^{2}(0, T, H)}$ and $\left\|g_{o p_{h}}\right\|_{L^{2}(0, T, H)}$ are also bounded independently of $h$. So there exists $f$ and $\eta$ in $L^{2}(0, T, H)$ such that

$$
g_{o p_{h}} \rightarrow f \quad \text { in } \quad L^{2}(0, T, H) \quad(\text { weak }) \quad \text { and } \quad u_{g_{o p_{h}}} \rightarrow \eta \quad \text { in } \quad L^{2}(0, T, H) \quad \text { (weak). (3.10) }
$$

Taking now $v=u_{g_{o p}}(t) \in K$ in (2.1), for $\left.t \in\right] 0, T$, with $u=u_{g_{o p_{h}} h}$ and $g=g_{o p_{h}}$, we obtain

$$
\begin{array}{r}
\left\langle\dot{u}_{g_{o p_{h}} h}, u_{g_{o p}}-u_{g_{o p_{h}} h}\right\rangle+a_{1}\left(u_{g_{o p_{h}} h}, u_{g_{o p}}-u_{g_{o p_{h} h}}\right) \\
+(h-1) \int_{\Gamma_{1}} u_{g_{o p_{h} h}}\left(u_{g_{o p}}-u_{g_{o p_{h} h}}\right) d s+\Phi\left(u_{g_{o p}}\right)-\Phi\left(u g_{o p_{h} h}\right) \geq \\
\left.\left(g_{o p_{h}}, u_{g_{o p}}-u_{g_{o p_{h}} h}\right)+h \int_{\Gamma_{1}} b\left(u_{g_{o p}}-u_{g_{o p_{h}} h}\right) d s, \quad \text { a.e.t } \in\right] 0, T[
\end{array}
$$

as $u_{g_{o p}}=b$ on $\Gamma_{1} \times[0, T]$, taking $u_{g_{o p}}-u_{g_{o p_{h}} h}=\phi_{h}$ we obtain

$$
\begin{aligned}
& \left\langle\dot{\phi}_{h}, \phi_{h}\right\rangle+a_{1}\left(\phi_{h}, \phi_{h}\right)+(h-1) \int_{\Gamma_{1}}\left|\phi_{h}\right|^{2} d s \leq-\left(g_{o p_{h}}, \phi_{h}\right) \\
& \left.\quad+\int_{\Gamma_{2}} q\left|\phi_{h}\right| d s+\left\langle\dot{u}_{g_{o p}}, \phi_{h}\right\rangle+a\left(u_{g_{o p}}, \phi_{h}\right), \quad \text { a.e. } t \in\right] 0, T[
\end{aligned}
$$

then

$$
\begin{array}{r}
\frac{1}{2}\left\|\phi_{h}\right\|_{L^{\infty}(0, T, H)}^{2}+\lambda_{1}\left\|\phi_{h}\right\|_{L^{2}(0, T, V)}^{2}+(h-1) \int_{0}^{T} \int_{\Gamma_{1}}\left|\phi_{h}(t)\right|^{2} d s d t \\
\leq-\int_{0}^{T}\left(g_{o p_{h}}(t), \phi_{h}(t)\right) d t+\int_{0}^{T} \int_{\Gamma_{2}} q\left|\phi_{h}(t)\right| d s d t+\int_{0}^{T}\left\langle\dot{u}_{g_{o p}}(t), \phi_{h}(t)\right\rangle d t \\
+\int_{0}^{T} a\left(u_{g_{o p_{h}} h}(t), \phi_{h}(t)\right) d t .
\end{array}
$$


There exists a constant $C>$ which does not depend on $h$ such that

$$
\begin{array}{r}
\left\|\phi_{h}\right\|_{L^{2}(0, T, V)}=\left\|u_{g_{o p_{h}} h}-u_{g_{o p}}\right\|_{L^{2}(0, T, V)} \leq C, \quad\left\|\phi_{h}\right\|_{L^{\infty}(0, T, H)} \leq C \\
\text { and }(h-1) \int_{0}^{T} \int_{\Gamma_{1}}\left|u_{g_{o p_{h}} h}-b\right|^{2} d s d t \leq C,
\end{array}
$$

then $\eta \in L^{2}(0, T, V)$ and

$$
\begin{gathered}
u_{g_{o p_{h} h}} \rightarrow \eta \quad \text { in } L^{2}(0, T, V) \quad \text { weak and in } L^{\infty}(0, T, H) \text { weak star } \\
u_{g_{o p_{h} h}} \rightarrow b \quad \text { in } L^{2}\left(0, T, L^{2}\left(\Gamma_{1}\right)\right) \text { strong, }
\end{gathered}
$$

so $\eta(t) \in K$ for all $t \in[0, T]$. Now taking $v \in K$ in (2.1) where $u=u_{g_{o p_{h}} h}$ and $g=g_{o p_{h}}$ so

$$
\begin{aligned}
\left\langle\dot{u}_{g_{o p_{h}}}, v-u_{g_{o p_{h} h}}\right\rangle+a_{h}\left(u_{g_{o p_{h}} h}, v-u_{g_{o p_{h} h}}\right) & +\Phi(v)-\Phi\left(u_{g_{o p_{h} h}}\right) \geq\left(g_{o p_{h}}, v-u_{g_{o p_{h}} h}\right) \\
& \left.+h \int_{\Gamma_{1}} b\left(v-u_{g_{o p_{h}} h}\right) d s, \quad \text { a.e. } t \in\right] 0, T[
\end{aligned}
$$

as $v \in K$ so $v=b$ on $\Gamma_{1}$, thus we have

$$
\begin{aligned}
& \left\langle\dot{u}_{g_{o p_{h} h}}, u_{g_{o p_{h} h}}-v\right\rangle+a\left(u_{g_{o p_{h} h}}, u_{g_{o p_{h} h}}-v\right)+h \int_{\Gamma_{1}}\left|u_{g_{o p_{h} h}}-b\right|^{2} d s+\Phi\left(u_{g_{o p_{h} h}}\right)-\Phi(v) \\
& \leq\left\langle-\left(g_{o p_{h}}, v-u_{g_{o p_{h} h}}\right) \quad \text { a.e. } t \in\right] 0, T[\text {. }
\end{aligned}
$$

Thus

$$
\begin{array}{r}
\left\langle\dot{u}_{g_{o p_{h} h}}, u_{g_{o p_{h} h}}-v\right\rangle+a\left(u_{g_{o p_{h} h}}, u_{g_{o p_{h} h}}-v\right)+\Phi\left(u_{g_{o p_{h} h}}\right)-\Phi(v) \leq-\left(g_{o p_{h}}, v-u_{g_{o p_{h} h}}\right) \\
\text { a.e. } t \in] 0, T[.
\end{array}
$$

Using (3.10) and (3.11) and the same arguments as in (2.14)- (2.19), we deduce that

$$
\langle\dot{\eta}, v-\eta\rangle+a(\eta, v-\eta)+\Phi(v)-\Phi(\eta) \geq(f, v-\eta), \quad \forall v \in K, \quad \text { a.e.t } \in] 0, T[,
$$

so also by the uniqueness of the solution of (1.1) we obtain that

$$
u_{f}=\eta
$$

We prove that $f=g_{o p}$. Indeed we have

$$
\begin{aligned}
J(f) & =\frac{1}{2}\|\eta\|_{L^{2}(0, T ; H)}^{2}+\frac{M}{2}\|f\|_{L^{2}(0, T ; H)}^{2} \\
& \leq \liminf _{h \rightarrow+\infty}\left\{\frac{1}{2}\left\|u_{g_{o p_{h}} h}\right\|_{L^{2}(0, T ; H)}^{2}+\frac{M}{2}\left\|g_{o p_{h}}\right\|_{L^{2}(0, T ; H)}^{2}\right\}=\liminf _{h \rightarrow+\infty} J_{h}\left(g_{o p_{h}}\right) \\
& \leq \liminf _{h \rightarrow+\infty} J_{h}(g)=\liminf _{h \rightarrow+\infty}\left\{\frac{1}{2}\left\|u_{g_{h}}\right\|_{L^{2}(0, T ; H)}^{2}+\frac{M}{2}\|g\|_{L^{2}(0, T ; H)}^{2}\right\}
\end{aligned}
$$

using now the strong convergence $u_{g_{h}} \rightarrow u_{g}$ as $h \rightarrow+\infty, \forall g \in H$ (see Lemma 3.2), we obtain that

$$
J(f) \leq \liminf _{h \rightarrow+\infty} J_{h}\left(g_{o p_{h}}\right) \leq \frac{1}{2}\left\|u_{g}\right\|_{L^{2}(0, T ; H)}^{2}+\frac{M}{2}\|g\|_{L^{2}(0, T ; H)}^{2}=J(g), \quad \forall g \in L^{2}(0, T ; H)(3.1
$$


then by the uniqueness of the optimal control problem (1.9) we get

$$
f=g_{o p}
$$

Now we prove the strong convergence of $u_{g_{o p_{h}} h}$ to $\eta=u_{f}$ in $L^{2}(0, T, V) \cap L^{\infty}(0, T, H) \cap$ $L^{2}\left(0, T, L^{2}\left(\Gamma_{1}\right)\right)$, indeed taking $v=\eta$ in (2.1) where $u=u_{g_{o p_{h}} h}$ and $g=g_{o p_{h}}$, as $\eta(t) \in K$ for $t \in[0, T]$, so $\eta=b$ on $\Gamma_{1}$, we obtain we get

$$
\begin{array}{r}
\left\langle\dot{u}_{g_{o p_{h} h}}-\dot{\eta}, u_{g_{o p_{h} h}}-\eta\right\rangle+a_{1}\left(u_{g_{o p_{h} h}}-\eta, u_{g_{o p_{h} h}}-\eta\right)+(h-1) \int_{\Gamma_{1}}\left|u_{g_{o p_{h} h}}-\eta\right|^{2} d s \\
+\Phi\left(u_{g_{o p_{h} h}}\right)-\Phi(\eta) \leq\left(g_{o p_{h}}, u_{g_{o p_{h} h}}-\eta\right)+\left\langle\dot{\eta}, u_{g_{o p_{h} h}}-\eta\right\rangle+a\left(\eta, u_{g_{o p_{h} h}}-\eta\right)
\end{array}
$$

thus

$$
\begin{array}{r}
\frac{1}{2}\left\|u_{g_{o p_{h} h}}-\eta\right\|_{L^{\infty}(0, T ; H)}^{2}+\lambda_{1}\left\|u_{g_{o p_{h} h}}-\eta\right\|_{L^{2}(0, T, V)}^{2} \\
+\int_{0}^{T}\left\{\Phi\left(u_{g_{o p_{h} h}}\right)-\Phi(\eta)\right\} d t+(h-1)\left\|u_{g_{o p_{h} h}}-\eta\right\|_{L^{2}\left(0, T, L^{2}\left(\Gamma_{1}\right)\right)}^{2} \\
\leq \int_{0}^{T}\left(g_{o p_{h}}(t), u_{g_{o p_{h} h}}(t)-\eta(t)\right) d t+\int_{0}^{T}\left\langle\dot{\eta}, u_{g_{o p_{h} h}}-\eta\right\rangle d t \\
+\int_{0}^{T} a\left(\eta(t), \eta(t)-u_{g_{o p_{h} h}}(t)\right) d t
\end{array}
$$

Using (3.11) and the weak semi-continuity of $\Phi$ we deduce that

$$
\begin{aligned}
\lim _{h \rightarrow+\infty}\left\|u_{g_{o p_{h}}}-\eta\right\|_{L^{\infty}(0, T ; H)} & =\lim _{h \rightarrow+\infty}\left\|u_{g_{o p_{h}} h}-\eta\right\|_{L^{2}(0, T, V)} \\
& =\left\|u_{g_{o p_{h} h}}-\eta\right\|_{L^{2}\left(0, T, L^{2}\left(\Gamma_{1}\right)\right)}=0
\end{aligned}
$$

and with (3.13) and (3.15) we deduce (3.7). As $f \in L^{2}(0, T, H)$, then from (3.14) with $g=f$ and (3.15) we can write

$$
\begin{aligned}
J(f) & =J\left(g_{o p}\right)=\frac{1}{2}\left\|u_{g_{o p}}\right\|_{L^{2}(0, T, H)}^{2}+\frac{M}{2}\left\|g_{o p}\right\|_{L^{2}(0, T, H)}^{2} \\
& \leq \liminf _{h \rightarrow+\infty} J_{h}\left(g_{o p_{h}}\right)=\liminf _{h \rightarrow+\infty}\left\{\frac{1}{2}\left\|u_{g_{o p_{h}}}\right\|_{L^{2}(0, T, H)}^{2}+\frac{M}{2}\left\|g_{o p_{h}}\right\|_{L^{2}(0, T, H)}^{2}\right\} \\
& \leq \lim _{h \rightarrow+\infty} J_{h}\left(g_{o p}\right)=J\left(\left(g_{o p}\right)\right.
\end{aligned}
$$

and using the strong convergence (3.7), we get

$$
\lim _{h \rightarrow+\infty}\left\|g_{o p_{h}}\right\|_{L^{2}(0, T, H)}=\left\|g_{o p}\right\|_{L^{2}(0, T, H)}
$$

Finally as

$$
\left\|g_{o p_{h}}-g_{o p}\right\|_{L^{2}(0, T ; H)}^{2}=\left\|g_{o p_{h}}\right\|_{L^{2}(0, T ; H)}^{2}+\left\|g_{o p}\right\|_{L^{2}(0, T ; H)}^{2}-2\left(g_{o p_{h}}, g_{o p}\right)
$$

and by the first part of (3.10) we have

$$
\lim _{h \rightarrow+\infty}\left(g_{o p_{h}}, g_{o p}\right)=\left\|g_{o p}\right\|_{L^{2}(0, T, H)}^{2}
$$

so from (3.17) and (3.18) we get (3.8). This ends the proof.

Acknowledgements: This work was realized while the second author was a visitor at Saint Etienne University (France) and he is grateful to this institution for its hospitality. 


\section{References}

[1] K. Ait HAdi, Optimal control of the obstacle problem: optimality conditions. IMA J. Math. Control Inform. 23 (2006), pp. 325-334.

[2] A. Amassad, D. Chenais and C. Fabre, Optimal control of an elastic contact problem involving Tresca friction law. Nonlinear Analysis 48 (2002), pp. 1107-1135

[3] N. Arada, H. El Fekih and J.P.Raymont, Asymptotic analysis of some control problems. Asymptotic Analysis 24 (2000), pp. 343-366.

[4] V. Barbu, Optimal control of variational inequalities. Research Notes in Mathematics, 100. Pitman (Advanced Publishing Program), Boston, MA, 1984.

[5] V. Barbu, Optimal control for free boundary problems. Confer. Sem. Mat. Univ. Bari No. 206 (1985).

[6] F. Ben Belgacem, H. El Fekih and J.P.Raymont, A penalized Robin approach for solving a parabolic equation with nonsmooth Dirichlet boundary conditions. Asymptotic Analysis 34 (2003), pp. 121-136.

[7] M. Bergounioux, Optimal control of an obstacle problem. Appl. Math. Optim. 36 (1997), pp. 147-172.

[8] M. Bergounioux, Use of augmented Lagrangian methods for the optimal control of obstacle problems. J. Optim. Th. Appl. 95 (1) (1997), pp. 101-126.

[9] M. Bergounioux And F. Mignot, Optimal control of obstacle problems: existence of Lagrange multipliers. ESAIM: Control, Optimisation and Calculus of Variations 5 (2000), pp. 45-70.

[10] M. Bergounioux And N. Merabet, Sensibility analysis for optimal control of problems governed by semilinear parabolic equations. Control and Cybernetics 29 (2000), pp. 861-886.

[11] M. Bergounioux And F. Tröltzsch, Optimal control of semilinear parabolic equations with state-constraints of Bottleneck type. ESAIM: Control, Optimisation and Calculus of Variations 4 (1999), pp. 595-608.

[12] J.F. Bonnans And E. CASAS, Quelques methodes pour le controle optimal de problèmes comportant des contraintes sur l'état. Analele Stiintifice ale Univ. Al. I Cuza din Iasi Tomul XXXII, s.I a, Matematicã, 32 (1986), pp. 57-62.

[13] M. Boukrouche And I. Ciuperca Asymptotic behaviour of solutions of lubrication problem in a thin domain with a rough boundary and Tresca fluid-solid interface law. Quart. Appl. Math. 64 (2006), pp. 561-591.

[14] M. Boukrouche And R. El Mir Non-isothermal, non-Newtonian lubrication problem with Tresca fluid-solid law. Existence and asymptotic of weak solutions. Nonlinear Anal. Real World Applications 9 (2008), pp. 674-692.

[15] M. Boukrouche And F. SAIDi Non-isothermal lubrication problem with Tresca fluidsolid interface law. Partie I. Nonlinear Anal. Real World Applications 7 (2006), pp. 1145-1166. 
[16] M. Boukrouche And D. A. TArzia, On a convex combination of solutions to elliptic variational inequalities. Electro. J. Differential Equations 2007(2007), No. 31, pp. 1-10.

[17] H. BRÉZIS, Equations et inéquations non linéaires dans les espaces vectoriels en dualité. Annales Inst. Fourier, 18 (1968), pp. 115-175.

[18] H. Brézis, Problèmes unilatéraux. J.Math. Pure et Appl. 51 (1972), pp. 1-162.

[19] A. Capatina, Optimal Control of a Signorini contact problem, Numer. Funct. Anal. Optimiz. 21 (2000), pp. 817-828

[20] H.S. Carslaw And J.C. Jaeger Conduction of heat in solids. Clarendon Press, Oxford (1959).

[21] M. Chipot, Elements of Nonlinear Analysis, Birkhäuzer Advanced Texts, 2000.

[22] G. Duvaut and J.L. Lions, Les Inéquations en Mécanique et en Physique. Dunod, 1972 .

[23] C.M. Gariboldi And D.A. TARzia, Convergence of distributed optimal controls on the internal energy in mixed elliptic problems when the heat transfer coefficient goes to infinity. Appl. Math. Optim. 47 (3) (2003), pp. 213-230.

[24] C.M. Gariboldi And D.A. Tarzia, Convergence of boundary optimal control problems with restrictions in mixed elliptic Stefan-like problems. Advances in Differential Equations and Control Processes, 1 (2008), pp. 113-132.

[25] K. Ito And K. Kunisch, Optimal control of elliptic variational inequalities. Appl. Math. Optim. 41 (2000), pp. 343-364.

[26] S. Kesavan and T. Muthukumar, Low-cost control problems on perforated and nonperforated domains, Proc. Indian Acad. Sci. (Math. Sci.) 118, No. 1, (2008) pp. 133-157.

[27] J.L. Lions, Contrôle optimal de systèmes gouvernés par des équations aux dérivées partielles. Dunod Paris (1968).

[28] J.L. Menaldi and D. A. Tarzia, A distributed parabolic control with mixed boundary conditions. Asymptotic Analysis 52 (2007), pp. 227-241.

[29] F. Mignot, Contrôle dans les inéquations variationelles elliptiques. J. Functional Analysis 22 (1976), no. 2, pp. 130-185.

[30] F. Mignot And J.P. Puel, Optimal control in some variational inequalities. SIAM J. Control Optim. 22 (3) (1984), pp. 466-476.

[31] P. Neittaanmaki, J. Sprekels and D. Tiba, Optimization of elliptic systems. Theory and applications. Springer Monographs in Mathematics. Springer, New York, 2006.

[32] P. Neittannmaki And D. TiBa, A variational Inequality approach to Constrained Control problems for parabolic equations. Appl. Math. Optim. 17 (1988), pp. 185-201.

[33] P. Neittaanmaki, and D. Tiba, Optimal control of nonlinear parabolic systems. Theory, algorithms and applications. Marcel Dekker Inc., New York, 1994.

[34] P. Neittaanmaki, J. Sprekels, D. Tiba, Optimization of elliptic systems. Theory and Applicatioins. Springer-Verlag, New York, 2006. 
[35] O. Pironneau, Optimal shape design for elliptic systems Springer-Verlag, New York, 1984.

[36] E.D. TABACMAN AND D. A. TARZIA, Sufficient and/or necessary condition for the heat transfer coefficient on $\Gamma_{1}$ and the heat flux on $\Gamma_{2}$ to obtain a steady-state two-phase Stefan problem. J. Differential Equations 77 (1989), pp. 16-37.

[37] D. A. TARzIA, Una familia de problemas que converge hacia el caso estacionario del problema de Stefan a dos fases, Math. Notae 27 (1979), pp. 157-165.

[38] D. A. TARzIA, Etude de l'inéquation variationnelle proposée par Duvaut pour le problème de Stefan à deux phases, I, Boll. Unione Mat. Italiana 1B (1982), pp. 865883.

[39] Y. Ye And Q. Chen, Optimal control of the obstacle problem in a quasilinear elliptic variational inequality. J. Math. Anal. Appl. 294 (2004), pp. 258-272. 